\title{
A new multi-criteria decision-making method based on Pythagorean hesitant fuzzy Archimedean Muirhead mean operators $^{1}$
}

\author{
Yanru Zhong ${ }^{\mathrm{a}}$, Xiuyan Guo ${ }^{\mathrm{a}}$, Hong Gao ${ }^{\mathrm{a}}$, Yuchu Qin ${ }^{\mathrm{b}}$, Meifa Huang ${ }^{\mathrm{c}, *}$ and Xiaonan Luo ${ }^{\mathrm{a}}$ \\ ${ }^{a}$ Guangxi Key Laboratory of Intelligent Processing of Computer Images and Graphic, Guilin University of \\ Electronic Technology, Guilin, PR China \\ ${ }^{\mathrm{b}}$ School of Computing and Engineering, University of Huddersfield, Huddersfield, United Kingdom \\ c School of Mechanical and Electrical Engineering, Guilin University of Electronic Technology, Guilin, PR China
}

\begin{abstract}
To describe the values of criteria and to generate a sort of alternatives are two important issues in multi-criteria decision-making (MCDM). A superior tool for the former issue is Pythagorean hesitant fuzzy number (PHFN) and an effective tool for the latter issue is aggregation operator. So far, a number of aggregation operators of PHFNs have been presented within the academia. Each aggregation operator has its own characteristics and can work well for its specific purpose. But there is not yet an aggregation operator of PHFNs that can provide satisfying generality and flexibility in aggregating the values of criteria and capturing the interactions of criteria. The Archimedean t-conorm and t-norm (ATT) are well-known for having the capability to generate versatile and flexible operational rules for fuzzy numbers, while the Muirhead mean (MM) operator is an all-in-one aggregation operator for capturing the interrelationships of the aggregated arguments. To this end, the MM operator and the ATT for PHFNs are combined to present a Pythagorean hesitant fuzzy Archimedean MM (PHFAMM) operator and a weighted PHFAMM operator and a new MCDM method based on the presented operators is proposed in this paper. Firstly, the generalised expressions of the presented operators are provided. The properties of the operators are explored and proved and their specific expressions based on Algebraic, Einstein, Hamacher, and Frank ATTs are then constructed. Based on these specific expressions, a new method for solving the MCDM problems based on PHFNs is developed. Finally, the developed MCDM method is demonstrated via an example, a set of experiments and qualitative and quantitative comparisons.
\end{abstract}

Keywords: Pythagorean hesitant fuzzy set, Muirhead mean operator, Archimedean t-conorm and t-norm, Aggregation operator, Multi-criteria decision-making

\section{Introduction}

Multi-criteria decision-making (MCDM) is a process of finding desirable alternatives via evaluating the values of multiple criteria of all alternatives synthetically [1]. In this process, a primary issue is to describe the values of criteria. For this issue, many experts proposed to adopt fuzzy sets [2]. For example, Atanassov [3-5] introduced the intuitionistic fuzzy set (IFS), which is a generalization of fuzzy set. Garg and Rani $[6,7]$ extended the aggregation operators for IFS to deal with two-dimensional information in a set. Yager $[8,9]$ introduced the Pythagorean fuzzy set (PFS), which improves the expressiveness of IFS. Zhang and $\mathrm{Xu}$ [10] developed a set of new operational laws for PFSs and explored their desirable properties. Garg [11-14] improved the existing aggregation operators for PFSs by adding the pairs of the hesitation between the membership functions and proposed some new operational laws for Pythagorean fuzzy numbers using Einstein operations. Gou et al. [15] studied the continuity, derivability, and differentiability of continuous Pythagorean fuzzy information. Peng [16] extended the scope of PFS to combine soft set with rough set, employ PFS in the information environment of lan-

\footnotetext{
${ }^{1}$ This research was funded by the National Natural Science Foundation of China (No.61562016; No.61772149; No.61762028), Guangxi Key Laboratory of Images and Graphics Intelligent Processing (No.GIIP1805), and the Innovation Key Project of Guangxi Province (No. AA18118039-2).

*Corresponding author. Meifa Huang. E-mail: meifahuang@yeah.net (MH).
} 
guage and propose a method to manage the membership and non-membership degrees expressed by interval-values. Garg [17] presented the linguistic Pythagorean fuzzy set by combining PFSs and linguistic fuzzy sets. Wei and Lu [18] proposed a set of Pythagorean fuzzy power aggregation operators and studied the prominent characteristics of these operators. By aggregated interval-valued Pythagorean fuzzy set (IVPFS) and the Maclaurin symmetric mean (MSM) operator, Garg [19] explored the IVPFSMSM operators and their weighted forms for solving MCDM problem, which can consider the interaction between the pairs of the membership degrees. In addition, Garg $[20,21]$ also developed two new exponential operational laws and the aggregation operators to cope with crisp values on IVPFS in MCDM. Peng [22,23] proposed a novel score function, a new distance measure with multiple parameters and novel algorithms for IVPFS in the context of MCDM. Torra and Narukawa $[24,25]$ presented the concept of hesitant fuzzy set (HFS), which allows the membership degree of an element to be a set of some feasible values between 0 and 1. Qin et al. [26] investigated the Frank triangular norms with hesitant fuzzy information. Xu and Liang [27] proposed a distance measure for Pythagorean hesitant fuzzy sets (PHFSs), which can consider the differences of the membership, non-membership, and indeterminacy degrees.

Another important issue in MCDM is to generate a sort of alternatives. For this issue, there are normally two categories of approaches. One category is comprised by conventional approaches (e.g. TOPSIS, VIKOR, PROMETHEE, ELECTRE). The other category consists of the approaches based on aggregation operators. Aggregation operators can deal with the MCDM problems more effectively because they can provide comprehensive values and ranking of alternatives, whereas conventional approaches can only generate ranking [28]. Recently, a number of different aggregation operators for PHFSs have been presented, which mainly include the weighted average and geometric operators [29], Hamacher aggregation operators [30], MSM operator [31], Bonferroni mean (BM) operator [32], Hybrid aggregation operator [33], Dual hesitant Pythagorean fuzzy Hamacher operator [34], and Archimedean aggregation operators [35]. Among these operators, the Archimedean Pythagorean hesitant fuzzy weighted averaging operator and weighted geometric operator [35] are the most generalized aggregation operators of Pythagorean hesitant fuzzy numbers (PHFNs). In them, the operations are performed on the basis of any types of Archimedean tconorm and t-norm (ATT) (e.g. Algebraic, Einstein,
Hamacher, and Frank t-conorms and t-norms) [36-38]. Due to such characteristic, the operators are more generalized, versatile and flexible than those operators based on one specific t-conorm and t-norm.

In addition to the generality of the operations, the capability to capture the interactions among criteria is also an important feature of aggregation operators [39]. Among the existing aggregation operators of PHFNs, the MSM and BM operators have this capability. The Muirhead mean (MM) operator [40], a generalization of the arithmetic average, BM [41], MSM [42,43] and geometric average operators, also has such capability [44]. A number of researchers have explored the MM operator in various fuzzy environments for MCDM. For example, Liu and Li [45] developed an MM operator to aggregate hesitant fuzzy linguistic information. Wang J et al. [46] developed a hesitant fuzzy linguistic MM operator. Hong and Rong [44] presented a hesitant fuzzy dual Muirhead mean operator. Zhu and Li [47] proposed a Pythagorean fuzzy MM operator and a Pythagorean fuzzy dual MM operator.

Based on the considerations above, it is non-trivial to combine the MM operators with the ATTs under Pythagorean hesitant fuzzy environment. In this paper, such combination is studied in depth. The major contributions of the paper are to present and explore the properties of a Pythagorean hesitant fuzzy Archimedean MM (PHFAMM) operator and its weighted form and to propose a new method based on the presented operators to resolve the MCDM problems based on PHFNs. To the best of the knowledge, this is the first PHFNs based MCDM method that combines the MM operators and the ATTs in the context of PHFSs.

The remainder of the paper is organized as follows. Section 2 gives a brief introduction to some related concepts. Section 3 explains the details of the presented operators. Section 4 elaborates the proposed MCDM method. Section 5 demonstrates the proposed method via a practical example, a set of experiments, and comparisons with existing methods. Section 6 ends the paper with a conclusion.

\section{Preliminaries}

\subsection{Operator}

Definition 1. [40] Let $a_{i}(i=1,2, \ldots, n)$ be a collection of nonnegative real members, $A=\left\{a_{1}, a_{2}, \ldots, a_{n}\right\}$ and $P=\left(p_{1}, p_{2}, \ldots, p_{n}\right) \in R^{n}$ be a parameter vector, if 


$$
\begin{aligned}
& M^{P}\left(a_{1}, a_{2}, a_{3}, \ldots a_{n}\right) \\
& =\left(\frac{1}{n !}\left(\sum_{\theta \in S_{n}}\left(\prod_{j=1}^{n} a_{\theta(j)}^{p_{j}}\right)\right)\right)^{\frac{1}{\sum_{j=1}^{n} p_{j}}}
\end{aligned}
$$

then $\mathrm{MM}^{P}$ is called the MM operator, where $\vartheta_{(j)}(j=$ $1,2, \ldots, n)$ is a permutation of $(1,2, \ldots, n)$ and $S_{n}$ is the collection of all permutations of $\vartheta_{(j)}$.

An advantage of the MM operator is that it can capture the interrelationships among the multiple aggregated arguments. In addition, the MM operator is a generalization of a number of existing aggregation operators. The special cases of MM operator are as follows:

Case 1. If $P=(1,0, \ldots, 0)$, the MM operator will reduce to the arithmetic average operator:

$$
M M^{(1,0, \ldots, 0)}\left(a_{1}, a_{2}, \ldots, a_{n}\right)=\frac{1}{n} \sum_{j=1}^{n} a_{j}
$$

Case 2. If $P=\left(\frac{1}{n}, \frac{1}{n}, \ldots, \frac{1}{n}\right)$, the MM operator will reduce to the geometric average operator:

$$
M M^{\left(\frac{1}{n}, \frac{1}{n}, \ldots, \frac{1}{n}\right)}\left(a_{1}, a_{2}, \ldots, a_{n}\right)=\prod_{j=1}^{n} a_{j}^{\frac{1}{n}}
$$

Case 3. If $P=(1,1,0,0, \ldots, 0)$, the MM operator will reduce to the $\mathrm{BM}$ operator:

$$
M M^{(1,1,0,0 \ldots, \ldots)}\left(a_{1}, a_{2}, \ldots, a_{n}\right)=\left(\frac{1}{n(n-1)} \sum_{\substack{i, j=1 \\ j \neq i}}^{n} a_{i}^{p} a_{j}^{q}\right)^{\frac{1}{p+q}}
$$

Case 4. If $P=(\underbrace{1,1, \ldots 1}_{k}, \underbrace{0,0, \ldots 0}_{n-k})$, the MM operator will reduce to the MSM operator:

$$
M M \underbrace{(\underbrace{1,1, \ldots, 1,0, \ldots, 0)}_{k}, \ldots-k}\left(a_{1}, a_{2}, \ldots, a_{n}\right)=\left(\frac{\sum_{1 \leq i_{1}<\ldots<i_{k} \leq n} \prod_{j=1}^{k} a_{j}}{C_{n}^{k}}\right)^{\frac{1}{k}}
$$

\subsection{PHFS Theory}

Definition 2. [29] Let $X$ be a universe of discourse. A PHFS $P$ on $X$ is given by

$$
P=\left\{<x, h_{p}(x)>\mid x \in X\right\}
$$

where $h_{p}(x)=\{\mu(x), v(x)\}$ denotes a set of possible Pythagorean fuzzy numbers on $X$, and $\mu(x)$ and $v(x)$ denote the membership and non-membership degrees of the element $x \in X$ to the set $\mathrm{P}$, respectively. For convenience, $p=h_{p}(x)=\mathrm{U}_{(\gamma, \eta) \in h_{p}(x)}\{(\gamma, \eta)\} \quad(0 \leq$ $\gamma, \eta \leq 1$ and $\left.0 \leq \gamma^{2}+\eta^{2} \leq 1\right)$ is called a PHFN. A PHFN is generally simplified as $p=h_{p}=(\mu, v)$.

Definition 3. [30] For any PHFN $h_{p}=(\mu, v)$, the score function of $h_{p}$ is defined as follow:

$$
S(p)=\frac{1}{2}\left(1+\frac{1}{l_{h}} \sum_{(\gamma, \eta) \in(\mu, v)}\left(\gamma^{2}-\eta^{2}\right)\right)
$$

where $\mathrm{S}\left(h_{p}\right) \in[-1,1]$; For any $\operatorname{PHFN} h_{p}=(\mu, v)$, the accuracy function of $h_{p}$ is defined as follow:

$$
A(p)=\frac{1}{l_{h}} \sum_{(\gamma, \eta) \in(\mu, v)}\left(\gamma^{2}+\eta^{2}\right)
$$

where $l_{h}$ is the number of elements in $(\mu, v)$.

Definition 4. [10, 29] Let $h_{1 p}=\left(\mu_{1}, v_{1}\right)$ and $h_{2 p}=$ $\left(\mu_{2}, v_{2}\right)$ be any two PHFNs, $S\left(h_{i p}\right)$ is the score function and $A\left(h_{i p}\right)$ is accuracy function of $h_{i p}(i=1,2)$. Then the ordering of those two PHFNs are given by

(1) if $S\left(h_{1 p}\right)>S\left(h_{2 p}\right)$, then $h_{1 p}$ is superior to $h_{2 p}$, denoted by $h_{1 p}>h_{2 p}$;

(2) if $S\left(h_{1 p}\right)=S\left(h_{2 p}\right)$, then

a. if $A\left(h_{1 p}\right)>A\left(h_{2 p}\right)$, then $h_{1 p}>h_{2 p}$

b. if $A\left(h_{1 p}\right)=A\left(h_{2 p}\right)$, then $h_{1 p}$ is equivalent to $h_{2 p}$, denoted by $h_{1 p} \approx h_{2 p}$.

\subsection{Operational Laws of PHFNs Based on ATTs}

Definition 5. [35] A fuzzy t-conorm is a binary operation $U:[0,1] \times[0,1] \rightarrow[0,1]$ that satisfies the following conditions for all $a, b, d \in[0,1]$ :

(1) $U(a, 0)=a$ for all $a$.

(2) $U(a, b)=U(b, a)$ for all $a$ and $b$.

(3) If $b<b^{*}$ and $d<d^{*}$ then $U(b, d) \leq U\left(b^{*}, d^{*}\right)$.

(4) $U(a, U(b, d))=U(U(a, b), d)$ for all $a, b$ and $d$.

Definition 6. [35] A fuzzy t-norm is a binary operation $I:[0,1] \times[0,1] \rightarrow[0,1]$ that satisfies the following conditions for all , $b, d \in[0,1]$ :

(1) $I(a, 1)=a$ for all $a$.

(2) $I(a, b)=I(b, a)$ for all $a$ and $b$.

(3) If $b<b^{*}$ and $d<d^{*}$ then $I(b, d) \leq I\left(b^{*}, d^{*}\right)$.

(4) $I(a, I(b, d))=I(I(a, b), d)$ for all $a, b$ and $d$.

Definition 7. [35] A fuzzy t-norm $I(a, b)$ is called Archimedean t-norm (ATN) if every sequence $x_{n}$ (where $n=1,2, \ldots ; x_{1}<1$; and $x_{n+1}=I\left(x_{n}, x_{n}\right)$ converges to 0 . The conorm of an ATN is called Archimedean tconorm (ATC).

For ATN and ATC:

(1) If $f(t)(t \in R)$ is monotonically decreasing and satisfies the conditions that $f(t):(0,1] \rightarrow$ $R^{+} ; f^{-1}(t): R^{+} \rightarrow(0,1] ; \lim _{t \rightarrow \infty} f^{-1}(t)=0$; $f^{-1}(0)=1$, then $f(t)$ can be used to express $I$ and is called the additive generator of $I$ :

$$
I(a, b)=f^{-1}(f(a)+f(b))
$$

for all $a, b \in[0,1]$.

(2) If $g(t)(t \in R)$ is monotonically increasing and satisfies the conditions that $g(t):(0,1] \rightarrow$ 
$R^{+} ; g^{-1}(t): R^{+} \rightarrow(0,1] ; \lim _{t \rightarrow \infty} g^{-1}(t)=1$; $g^{-1}(0)=0$, then $g(t)$ can be used to express $U$ and is called the additive generator of $U$ :

$$
U(a, b)=g^{-1}(g(a)+g(b))
$$

Please note that $g(t)=f(1-t)$ for all $a, b \in[0,1]$.
Four specific types of ATTs and their additive generators are shown in the Tables 1 and 2 [35]. From the two tables, it is not difficult to derive the inverses of the additive generators (i. e. $f^{-1}$ and $g^{-1}$ ) of each type of ATT. They are omitted here due to space limitations.

Table 1

Four types of ATNs and their additive generators

\begin{tabular}{ccc}
\hline Name & ATN & Additive generator $f(t)$ \\
\hline Algebraic & $I^{A}(a, b)=a b$ & $f(t)=-\ln t$ \\
Einstein & $I^{E}(a, b)=\frac{a b}{1+(1-a)(1-b)}$ & $f(t)=-\ln \frac{2-t}{t}$ \\
Hamacher & $I^{H}(a, b)=\frac{a b}{\theta+(1-\theta)(a+b-a b)}$, where $\theta>0$ & $f(t)=\ln \frac{\theta+(1-\theta) t}{t}$, where $\theta>0$ \\
Frank & $I^{F}(a, b)=\log _{\theta} 1+\frac{\left(\theta^{a}-1\right)\left(\theta^{b}-1\right)}{\theta-1}$, where $\theta>1$ & $f(t)=\ln \frac{\theta-1}{\theta^{t}-1}$, where $\theta>1$ \\
\hline
\end{tabular}

Table 2

Four types of ATCs and their additive generators

\begin{tabular}{ccc}
\hline Name & ATC & Additive generator $g(t)$ \\
\hline Algebraic & $U^{A}(a, b)=a+b-a b$ & $g(t)=-\ln (1-t)$ \\
Einstein & $U^{E}(a, b)=\frac{a+b}{1+a b}$ & $g(t)=\ln \frac{1+t}{1-t}$ \\
Hamacher & $U^{H}(a, b)=\frac{a+b-a b-(1-\theta) a b}{1-(1-\theta) a b}$, where $\theta>0$ & $g(t)=\ln \frac{\theta+(1-\theta)(1-t)}{1-t}$, where $\theta>0$ \\
Frank & $U^{F}(a, b)=1-\log _{\theta} 1+\frac{\left(\theta^{1-a}-1\right)\left(\theta^{1-b}-1\right)}{\theta-1}$, where $\theta>1$ & $g(t)=\ln \frac{\theta-1}{\theta^{1-t}-1}$, where $\theta>1$ \\
\hline
\end{tabular}

Definition 8. [35] Let $h_{p}=(\mu, v)$ and $\lambda>0$. Then there are two operational laws for the PHFNs based on ATT:

(1) $\lambda h_{p}=\bigcup_{(\gamma, \eta) \in(\mu, \nu)}\left\{\left(\sqrt{g^{-1}\left(\lambda g\left(\gamma^{2}\right)\right)}, \sqrt{f^{-1}\left(\lambda f\left(\eta^{2}\right)\right)}\right)\right\}$

(2) $h_{p}^{\lambda}=\bigcup_{(\gamma, \eta) \in(\mu, v)}\left\{\left(\sqrt{f^{-1}\left(\lambda f\left(\gamma^{2}\right)\right)}, \sqrt{g^{-1}\left(\lambda g\left(\eta^{2}\right)\right)}\right)\right\}$

Definition 9. [35] Let $h_{j p}=\left(u_{j}, v_{j}\right)(j=1,2, \ldots n)$ be a set of PHFNs, $w=\left(w_{1}, w_{2}, \ldots, w_{n}\right)^{T}$ be the weight vector of $h_{j p}$, where $w_{j} \in[0,1]$ and $\sum_{j=1}^{n} w_{j}=1$. An Archimedean Pythagorean hesitant fuzzy weighted average (APHFWA) operator is a mapping operation: $P^{n} \rightarrow \mathrm{P}$, defined by $\operatorname{APHFWA}\left(p_{1}, p_{2}, \ldots, p_{n}=\right.$ $\left.\bigoplus_{i=1}^{n}\left(w_{i} p_{i}\right)\right)$, where $\oplus$ conveys the addition of all elements in $h_{j p}$. Then the aggregated value of the APHFWA operator is also a PHFN, and

$$
\begin{aligned}
& \text { APHFWA }\left(h_{1 p}, h_{2 p}, \ldots, h_{n p}\right) \\
& =\bigcup_{\substack{\left(\gamma_{i}, \eta_{i}\right) \in\left(\mu_{i}, v_{i}\right) \\
i=1,2, n}} \frac{\left\{\left(\sqrt{g^{-1}\left(\sum_{i=1}^{n} w_{j} g\left(\gamma_{i}^{2}\right)\right)},\right.\right.}{\left.\sqrt{\left.f^{-1}\left(\sum_{i=1}^{n} w_{j} f\left(\eta_{i}^{2}\right)\right)\right)}\right\}}
\end{aligned}
$$

Definition 10. [35] Let $h_{j p}=\left(u_{j}, v_{j}\right)(j=1,2, \ldots n)$ be a set of PHFNs, $w=\left(w_{1}, w_{2}, \ldots, w_{n}\right)^{T}$ be the weight vector of $h_{j p}$, where $w_{j} \in[0,1]$ and $\sum_{j=1}^{n} w_{j}=1$. Then, an Archimedean Pythagorean hesitant fuzzy weighted geometric (APHFWG) operator is a mapping operation: $P^{n} \rightarrow \mathrm{P}$, defined by $\operatorname{APsHFWG}\left(p_{1}, p_{2}, \ldots, p_{n}=\bigotimes_{i=1}^{n}\left(p_{i}{ }^{w_{i}}\right)\right)$, where $\otimes$ conveys the multiplication of all elements in $h_{j p}$. Then the aggregated value of the APHFWG operator is also a PHFN, and

$$
\begin{aligned}
& \operatorname{APHFWG}\left(h_{1 p}, h_{2 p}, \ldots, h_{n p}\right) \\
& =\bigcup_{\substack{\left(\gamma_{i}, \eta_{i}\right) \in\left(\mu_{i}, v_{i}\right) \\
i=1,2, \ldots n}}\left\{\left(\sqrt{f^{-1}\left(\sum_{i=1}^{n} w_{j} f\left(\gamma_{i}^{2}\right)\right)},\right.\right. \\
& \left.\left.\sqrt{g^{-1}\left(\sum_{i=1}^{n} w_{j} g\left(\eta_{i}^{2}\right)\right)}\right)\right\}
\end{aligned}
$$

\section{PHFAMM Operators}

In this section, a PHFAMM operator and a weighted PHFAMM (WPHFAMM) operator are presented using the MM operator and the operational laws of PHFNs based on ATTs. The properties of the two operators are explored and proved and their specific expressions are derived. 


\subsection{PHFAMM Operator}

Definition 11. Let $Q=\left(q_{1}, q_{2}, \ldots, q_{n}\right) \in R^{n}$ be a parameter vector and $h_{j p}=\left(u_{j}, v_{j}\right)(j=1,2, \ldots n)$ be a set of PHFNs. If

$$
\begin{aligned}
& \operatorname{PHFAMM}^{Q}\left(h_{1 p}, h_{2 p}, \ldots h_{n p}\right) \\
& =\left(\frac{1}{n !}\left(\bigoplus_{\vartheta \in S_{n}} \bigotimes_{j=1}^{n}\left(h_{p \vartheta_{(j)}}{ }^{q_{j}}\right)\right)\right)^{\frac{1}{\sum_{j=1}^{n} q_{j}}}
\end{aligned}
$$

Then PHFAMM ${ }^{Q}$ is called the PHFAMM operator. In this equation, $\vartheta_{(j)}(j=1,2, \ldots n)$ is any a permutation of $(1,2, \ldots n), S_{n}$ is the collection of all permutations of $(1,2, \ldots n), \oplus$ and $\otimes$ convey the same meaning as they are in Definition 9 and Definition 10, respectively. Theorem 1. Let $h_{j p}=\left(u_{j}, v_{j}\right)(j=1,2, \ldots n)$ be a set of PHFNs, $Q=\left(q_{1}, q_{2}, \ldots, q_{n}\right) \in R^{n}$ be a parameter vector, $\vartheta_{(j)}(j=1,2, \ldots n)$ be a permutation of $(1,2, \ldots n)$, and $S_{n}$ be the collection of all permutations of $(1,2, \ldots n)$.Then $\operatorname{PHFAMM}^{Q}\left(h_{1 p}, h_{2 p}, \ldots h_{n p}\right)$ is still a PHFN, and

$$
\begin{aligned}
& \operatorname{PHFAMM}^{Q}\left(h_{1 p}, h_{2 p}, \ldots h_{n p}\right)
\end{aligned}
$$

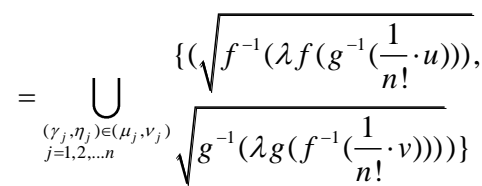

where, $\lambda=\frac{1}{\sum_{j=1}^{n} q_{j}}$,

$$
\begin{gathered}
u=\sum_{\theta=1}^{n} g\left(f^{-1}\left(\sum_{j=1}^{n} q_{j} f\left(\gamma_{\theta(j)}^{2}\right)\right)\right) \\
v=\sum_{\theta=1}^{n} f\left(g^{-1}\left(\sum_{j=1}^{n} q_{j} g\left(\eta_{\theta(j)}^{2}\right)\right)\right)
\end{gathered}
$$

Proof.

(1) Firstly, it is needed to prove that Equation (13) holds.

According to law (2) in Definition 8, it can be obtained that

$$
h_{p \vartheta_{(j)}}^{q_{j}}=\bigcup_{(\gamma, \eta) \in(\mu, \nu)}\left\{\left(\sqrt{f^{-1}\left(q_{j} f\left(\gamma^{2}\right)\right)}, \sqrt{g^{-1}\left(q_{j} g\left(\eta^{2}\right)\right)}\right)\right\}
$$

Obviously, $\Theta_{i=1}^{n} p_{i}$ can be derived from Equation (11)

$$
\otimes_{i=1}^{n} p_{i}=\bigcup_{\substack{\left(\gamma_{i}, \eta_{i}\right) \in\left(\mu_{i}, v_{i}\right) \\ i=1,2, \ldots n}}\left\{\left(\sqrt{f^{-1}\left(\sum_{i=1}^{n} f\left(\gamma_{i}^{2}\right)\right)},\right.\right.
$$

Thus, it is obtained that

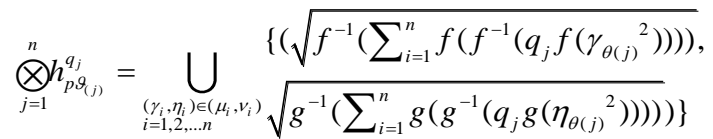

Obviously, $\oplus_{i=1}^{n} p_{i}$ can be derived from Equation (10)

$$
\oplus_{i=1}^{n} p_{i}=\bigcup_{\substack{\left(\gamma_{i}, \eta_{i}\right) \in\left(\mu_{i}, v_{i}\right) \\ i=1,2, \ldots n}}\left\{\left(\sqrt{g^{-1}\left(\sum_{i=1}^{n} g\left(\gamma_{i}^{2}\right)\right)},\right.\right.
$$

Thus, it is obtained that

$$
\begin{aligned}
& \bigoplus_{\vartheta \in S_{n}} \bigotimes_{j=1}^{n} h_{p \vartheta_{(j)}}^{q_{j}} \\
& =\bigcup_{\substack{\left(\gamma_{i}, \eta_{i}\right) \in\left(\mu_{i}, v_{i}\right) \\
i=1,2, \ldots n}}\left\{\left(\sqrt{g^{-1}\left(\sum_{\theta=1}^{n} g\left(f^{-1}\left(\sum_{i=1}^{n} f\left(f^{-1}\left(q_{j} f\left(\gamma_{\theta(j)}{ }^{2}\right)\right)\right)\right)\right)\right.}\right),\right.
\end{aligned}
$$

According to law (1) in Definition 8, it can be obtained that

$$
\begin{aligned}
& \frac{1}{n !}\left(\bigoplus_{\vartheta \in S_{n}} \bigotimes_{j=1}^{n} h_{p \vartheta_{(j)}}^{q_{j}}\right)
\end{aligned}
$$

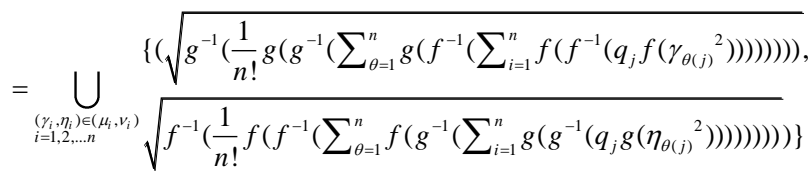

According to law (2) in Definition 8, the following equation is obtained:

$$
\begin{aligned}
& \left(\frac{1}{n !}\left(\bigoplus_{\vartheta \in S_{n}} \bigotimes_{j=1}^{n} h_{p \vartheta_{(j)}}^{q_{j}}\right)\right)^{\frac{1}{\sum_{j=1}^{n} q_{j}}} \\
& =\bigcup_{\substack{\left(\gamma_{j}, \eta_{j}\right) \in\left(\mu_{j}, v_{j}\right) \\
i=1,2, \ldots n}}\left\{\sqrt{f^{-1}\left(\lambda f\left(g^{-1}\left(\frac{1}{n !} \sum_{\theta=1}^{n} g\left(f^{-1}\left(\sum_{j=1}^{n} q_{j} f\left(\gamma_{\theta(j)}{ }^{2}\right)\right)\right)\right)\right)\right)},\right.
\end{aligned}
$$

where $\lambda=\frac{1}{\sum_{j=1}^{n} q_{j}}, \vartheta_{(j)}(j=1,2, \ldots n)$ is a permutation of $(1,2, \ldots n)$, and $S_{n}$ is the collection of all permutations of $(1,2, \ldots n)$. Thus, Equation (13) holds.

(2) The following will prove that Equation (13) is a PHFNs.

Let

$\mu_{h p}=\sqrt{f^{-1}\left(\lambda f\left(g^{-1}\left(\frac{1}{n !} \sum_{\theta=1}^{n} g\left(f^{-1}\left(\sum_{j=1}^{n} q_{j} f\left(\gamma_{\theta(j)}{ }^{2}\right)\right)\right)\right)\right)\right)}$

and

$$
v_{h p}=\sqrt{g^{-1}\left(\lambda g\left(f^{-1}\left(\frac{1}{n !} \sum_{\theta=1}^{n} f\left(g^{-1}\left(\sum_{j=1}^{n} q_{j} g\left(\eta_{\theta(j)}{ }^{2}\right)\right)\right)\right)\right)\right)}
$$

According to the definition of a PHFNs (Definition 2), it can be obtained that $0 \leq \gamma_{j} \leq 1$, $0 \leq \eta_{j} \leq 1$ and $0 \leq \gamma_{j}^{2}+\eta_{j}^{2} \leq 1$. Since $\vartheta_{(j)}(j=1,2, \ldots n)$ is a permutation of $(1,2, \ldots n)$ and $S_{n}$ is the collection of all permutations of $(1,2, \ldots n)$, the following inequalities are obtained according to the requirements of the additive generators $f(x)$ and $g(x)$ :

$0 \leq \mu_{h p} \leq 1,0 \leq v_{h p} \leq 1$. 
Thus, it is obtained that $0 \leq \gamma_{h p}^{2}+\eta_{h p}^{2} \leq 2$.

Since $\gamma_{\vartheta_{(j)}}^{2}+\eta_{\vartheta_{(j)}}^{2} \leq 1$, then $\gamma_{\vartheta_{(j)}}^{2} \leq 1-\eta_{\vartheta_{(j)}}^{2}$ and

$$
\begin{aligned}
& 0 \leq \mu_{h p}^{2}+v_{h p}^{2} \\
& =f^{-1}\left(\lambda f\left(g^{-1}\left(\frac{1}{n !} \sum_{\theta=1}^{n} g\left(f^{-1}\left(\sum_{j=1}^{n} q_{j} f\left(\gamma_{\theta(j)}^{2}\right)\right)\right)\right)\right)\right)+ \\
& g^{-1}\left(\lambda g\left(f^{-1}\left(\frac{1}{n !} \sum_{\theta=1}^{n} f\left(g^{-1}\left(\sum_{j=1}^{n} q_{j} g\left(\eta_{\theta(j)}^{2}\right)\right)\right)\right)\right)\right) \\
& \leq f^{-1}\left(\lambda f\left(g^{-1}\left(\frac{1}{n !} \sum_{\theta=1}^{n} g\left(f^{-1}\left(\sum_{j=1}^{n} q_{j} f\left(1-\eta_{\theta(j)}^{2}\right)\right)\right)\right)\right)+\right. \\
& g^{-1}\left(\lambda g\left(f^{-1}\left(\frac{1}{n !} \sum_{\theta=1}^{n} f\left(g^{-1}\left(\sum_{j=1}^{n} q_{j} g\left(\eta_{\theta(j)}^{2}\right)\right)\right)\right)\right)\right)
\end{aligned}
$$

Since $f\left(1-\eta_{\vartheta_{(j)}}^{2}\right)=g\left(\eta_{\vartheta_{(j)}}^{2}\right)$, it can be obtained that

$$
\begin{aligned}
& f^{-1}\left(\lambda f\left(g^{-1}\left(\frac{1}{n !} \sum_{\theta=1}^{n} g\left(f^{-1}\left(\sum_{j=1}^{n} q_{j} f\left(1-\eta_{\theta(j)}^{2}\right)\right)\right)\right)\right)\right)+ \\
& g^{-1}\left(\lambda g\left(f^{-1}\left(\frac{1}{n !} \sum_{\theta=1}^{n} f\left(g^{-1}\left(\sum_{j=1}^{n} q_{j} g\left(\eta_{\theta(j)}^{2}\right)\right)\right)\right)\right)\right)=1
\end{aligned}
$$

Therefore, the following inequality is attained:

$$
\begin{aligned}
& 0 \leq f^{-1}\left(\lambda f\left(g^{-1}\left(\frac{1}{n !} \sum_{\theta=1}^{n} g\left(f^{-1}\left(\sum_{j=1}^{n} q_{j} f\left(\gamma_{\theta(j)}^{2}\right)\right)\right)\right)\right)\right) \\
& +g^{-1}\left(\lambda g\left(f^{-1}\left(\frac{1}{n !} \sum_{\theta=1}^{n} f\left(g^{-1}\left(\sum_{j=1}^{n} q_{j} g\left(\eta_{\theta(j)}^{2}\right)\right)\right)\right)\right)\right) \leq 1
\end{aligned}
$$

That is, $0 \leq \mu_{h p}^{2}+v_{h p}^{2} \leq 1$.

According to the Definition 2, it can be concluded that the aggregated result of Equation (12) is a PHFN.

The following theorems state the idempotency, monotonicity and boundedness of the PHFAMM operator, respectively:

Theorem 2(Idempotency). Let $h_{j p}(j=1,2, \ldots n)$ (where $h_{j p}=\left(u_{j}, v_{j}\right)(j=1,2, \ldots n)$ ) be a collection of PHFNs and $Q=\left(q_{1}, q_{2}, \ldots, q_{n}\right) \in R^{n}$ be parameter vectors. If $h_{j p}(j=1,2, \ldots n)$ are equal, i.e., $h_{j p}=h_{p}$ (where $h_{p}=\left(u_{p}, v_{p}\right)$ ) for all $i$, then

$$
\operatorname{PHFAMM}^{Q}\left(h_{1 p}, h_{2 p}, \ldots h_{n p}\right)=h_{p}=\left(\mu_{p}, v_{p}\right)
$$

Proof.

According to the Theorem 1 yields

$$
\begin{aligned}
& \operatorname{PHFAMM}^{Q}\left(h_{1 p}, h_{2 p}, \ldots h_{n p}\right)
\end{aligned}
$$

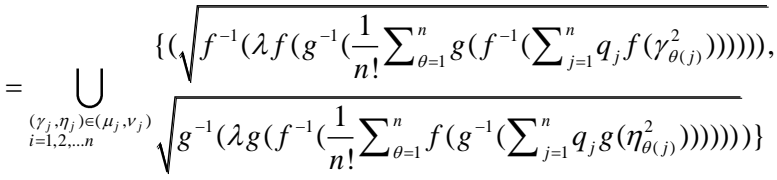

where $\lambda=\frac{1}{\sum_{j=1}^{n} q_{j}}$.

Since $h_{j p}=\bigcup_{\left(\gamma_{j}, \eta_{j}\right) \in\left(\mu_{j}, v_{j}\right)}\left\{\left(\gamma_{j}, \eta_{j}\right)\right\}=h_{p}=$ $\mathrm{U}_{(\gamma, \eta) \in(\mu, \nu)}\{(\gamma, \eta)\}$ i.e., $\gamma_{j}=\gamma$ and $\eta_{j}=\eta$, then $\gamma_{\theta(j)}^{2}=\gamma^{2}, \eta_{\theta(j)}^{2}=\eta^{2}$.

Then it can be achieved that $f\left(\gamma_{\theta(j)}^{2}\right)=f\left(\gamma^{2}\right), g\left(\eta_{\theta(j)}^{2}\right)=g\left(\eta^{2}\right)$

Then it is obtained that

$\sum_{j=1}^{n} q_{j} f\left(\gamma_{\theta(j)}^{2}\right)=\sum_{j=1}^{n} q_{j} \cdot f\left(\gamma^{2}\right)$,

$\sum_{j=1}^{n} q_{j} g\left(\eta_{\theta(j)}^{2}\right)=\sum_{j=1}^{n} q_{j} \cdot g\left(\eta^{2}\right)$.

and

$f^{-1}\left(\sum_{j=1}^{n} q_{j} f\left(\gamma_{\theta(j)}^{2}\right)\right)=f^{-1}\left(\sum_{j=1}^{n} q_{j} \cdot f\left(\gamma^{2}\right)\right)$,

$g^{-1}\left(\sum_{j=1}^{n} q_{j} g\left(\eta_{\theta(j)}^{2}\right)\right)=g^{-1}\left(\sum_{j=1}^{n} q_{j} \cdot g\left(\eta^{2}\right)\right)$,

Then it can be achieved that

$\sum_{\theta=1}^{n} g\left(f^{-1}\left(\sum_{j=1}^{n} q_{j} f\left(\gamma_{\theta(j)}^{2}\right)\right)\right)$

$=\sum_{\theta=1}^{n} g\left(f^{-1}\left(\sum_{j=1}^{n} q_{j} \cdot f\left(\gamma^{2}\right)\right)\right)$

$=n ! g\left(f^{-1}\left(\sum_{j=1}^{n} q_{j} \cdot f\left(\gamma^{2}\right)\right)\right)$,

$\sum_{\theta=1}^{n} f\left(g^{-1}\left(\sum_{j=1}^{n} q_{j} g\left(\eta_{\theta(j)}^{2}\right)\right)\right)$

$=\sum_{\theta=1}^{n} f\left(g^{-1}\left(\sum_{j=1}^{n} q_{j} \cdot g\left(\eta^{2}\right)\right)\right)$

$=n ! f\left(g^{-1}\left(\sum_{j=1}^{n} q_{j} \cdot g\left(\eta^{2}\right)\right)\right)$,

Then it is obtained that

$\frac{1}{n !} \sum_{\theta=1}^{n} g\left(f^{-1}\left(\sum_{j=1}^{n} q_{j} f\left(\gamma_{\theta(j)}^{2}\right)\right)\right)$

$=g\left(f^{-1}\left(\sum_{j=1}^{n} q_{j} \cdot f\left(\gamma^{2}\right)\right)\right)$,

$\frac{1}{n !} \sum_{\theta=1}^{n} f\left(g^{-1}\left(\sum_{j=1}^{n} q_{j} g\left(\eta_{\theta(j)}^{2}\right)\right)\right)$

$=f\left(g^{-1}\left(\sum_{j=1}^{n} q_{j} \cdot g\left(\eta^{2}\right)\right)\right)$,

Then it can be achieved that

$g^{-1}\left(\frac{1}{n !} \sum_{\theta=1}^{n} g\left(f^{-1}\left(\sum_{j=1}^{n} q_{j} f\left(\gamma_{\theta(j)}^{2}\right)\right)\right)\right)$

$=f^{-1}\left(\sum_{j=1}^{n} q_{j} \cdot f\left(\gamma^{2}\right)\right)$,

$f^{-1}\left(\frac{1}{n !} \sum_{\theta=1}^{n} f\left(g^{-1}\left(\sum_{j=1}^{n} q_{j} g\left(\eta_{\theta(j)}^{2}\right)\right)\right)\right)$

$=g^{-1}\left(\sum_{j=1}^{n} q_{j} \cdot g\left(\eta^{2}\right)\right)$,

Then it is obtained that

$f\left(g^{-1}\left(\frac{1}{n !} \sum_{\theta=1}^{n} g\left(f^{-1}\left(\sum_{j=1}^{n} q_{j} f\left(\gamma_{\theta(j)}^{2}\right)\right)\right)\right)\right)$

$=\sum_{j=1}^{n} q_{j} \cdot f\left(\gamma^{2}\right)$, 
$g\left(f^{-1}\left(\frac{1}{n !} \sum_{\theta=1}^{n} f\left(g^{-1}\left(\sum_{j=1}^{n} q_{j} g\left(\eta_{\theta(j)}^{2}\right)\right)\right)\right)\right)$

$=\sum_{j=1}^{n} q_{j} \cdot g\left(\eta^{2}\right)$,

Then it can be achieved that

$\lambda f\left(g^{-1}\left(\frac{1}{n !} \sum_{\theta=1}^{n} g\left(f^{-1}\left(\sum_{j=1}^{n} q_{j} f\left(\gamma_{\theta(j)}^{2}\right)\right)\right)\right)\right)$

$=\lambda \cdot \sum_{j=1}^{n} q_{j} \cdot f\left(\gamma^{2}\right)$,

$\lambda g\left(f^{-1}\left(\frac{1}{n !} \sum_{\theta=1}^{n} f\left(g^{-1}\left(\sum_{j=1}^{n} q_{j} g\left(\eta_{\theta(j)}^{2}\right)\right)\right)\right)\right)$

$=\lambda \cdot \sum_{j=1}^{n} q_{j} \cdot g\left(\eta^{2}\right)$,

where $\lambda=\frac{1}{\sum_{j=1}^{n} q_{j}}$. Then it is obtained that

$\lambda f\left(g^{-1}\left(\frac{1}{n !} \sum_{\theta=1}^{n} g\left(f^{-1}\left(\sum_{j=1}^{n} q_{j} f\left(\gamma_{\theta(j)}^{2}\right)\right)\right)\right)\right)$

$=f\left(\gamma^{2}\right)$,

$\lambda g\left(f^{-1}\left(\frac{1}{n !} \sum_{\theta=1}^{n} f\left(g^{-1}\left(\sum_{j=1}^{n} q_{j} g\left(\eta_{\theta(j)}^{2}\right)\right)\right)\right)\right)$

$=g\left(\eta^{2}\right)$,

Then it can be achieved that

$\sqrt{f^{-1}\left(\lambda f\left(g^{-1}\left(\frac{1}{n !} \sum_{\theta=1}^{n} g\left(f^{-1}\left(\sum_{j=1}^{n} q_{j} f\left(\gamma_{\theta(j)}^{2}\right)\right)\right)\right)\right)\right)}$

$=\sqrt{f^{-1}\left(f\left(\gamma^{2}\right)\right)}=\gamma$,

$\sqrt{g^{-1}\left(\lambda g\left(f^{-1}\left(\frac{1}{n !} \sum_{\theta=1}^{n} f\left(g^{-1}\left(\sum_{j=1}^{n} q_{j} g\left(\eta_{\theta(j)}^{2}\right)\right)\right)\right)\right)\right.}$

$=\sqrt{g^{-1}\left(g\left(\eta^{2}\right)\right)}=\eta$.

Finally, it is obtained that:

$\operatorname{PHFAMM}^{Q}\left(h_{1 p}, h_{2 p}, \ldots h_{n p}\right)=\left(\mu_{p}, v_{p}\right)$.

Theorem 3(Monotonicity). Let

$h_{j p}=\bigcup_{\substack{\left.\gamma_{j}, \eta_{j}\right) \in\left(\mu_{j}, v_{j}\right) \\ n=1,2, \ldots n}}\left\{\left(\gamma_{j}, \eta_{j}\right)\right\}$ and

$\tilde{h}_{j p}=\mathrm{U}_{\left(\widetilde{\gamma}_{j}, \tilde{\eta}_{j}\right) \in\left(\widetilde{\mu}_{j}, \widetilde{v}_{j}\right)}\left\{\left(\tilde{\gamma}_{j}, \tilde{\eta}_{j}\right)\right\}$ be two collections of $n=1,2, \ldots n$

PHFNs. For the PHFAMM ${ }^{\mathrm{Q}}$ operator, if $\gamma_{j} \geq$ $\widetilde{\gamma_{J}}$ and $\eta_{j} \geq \widetilde{\eta_{j}}$, then

$$
\begin{aligned}
& \operatorname{PHFAMM}^{Q}\left(h_{1 p}, h_{2 p}, \ldots h_{n p}\right) \geq \\
& \operatorname{PHFAMM}^{Q}\left(\tilde{h}_{1 p}, \tilde{h}_{2 p}, \ldots \tilde{h}_{n p}\right)
\end{aligned}
$$

Proof.

According to Theorem 1, it can be obtained that PHFAMM $^{Q}\left(h_{1 p}, h_{2 p}, \ldots h_{n p}\right)$

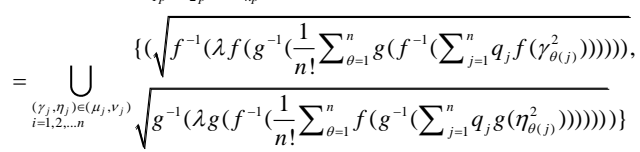

$\operatorname{PHFAMM}^{Q}\left(\tilde{h}_{1 p}, \tilde{h}_{2 p}, \ldots \tilde{h}_{n p}\right)$

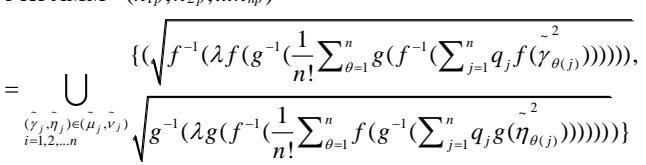

Since $f(x)$ and $f^{-1}(x)$ are monotonically decreasing, $g(x)$ and $g^{-1}(x)$ are monotonically increasing, $\gamma_{j} \geq$ $\tilde{\gamma}_{j}$ and $\eta_{j} \leq \tilde{\eta}_{j}$, it can be achieved that

$\sum_{j=1}^{n} q_{j} f\left(\gamma_{\theta(j)}^{2}\right) \leq \sum_{j=1}^{n} q_{j} f\left(\tilde{\gamma}_{\theta(j)}^{2}\right)$,

$\sum_{j=1}^{n} q_{j} g\left(\eta_{\theta(j)}^{2}\right) \leq \sum_{j=1}^{n} q_{j} g\left(\tilde{\eta}_{\theta(j)}^{2}\right)$, and

$f^{-1}\left(\sum_{j=1}^{n} q_{j} f\left(\gamma_{\theta(j)}^{2}\right)\right) \geq f^{-1}\left(\sum_{j=1}^{n} q_{j} f\left(\tilde{\gamma}_{\theta(j)}^{2}\right)\right)$,

$g^{-1}\left(\sum_{j=1}^{n} q_{j} g\left(\eta_{\theta(j)}^{2}\right)\right) \leq g^{-1}\left(\sum_{j=1}^{n} q_{j} g\left(\tilde{\eta}_{\theta(j)}^{2}\right)\right)$.

Then it is obtained that

$g\left(f^{-1}\left(\sum_{j=1}^{n} q_{j} f\left(\gamma_{\theta(j)}^{2}\right)\right)\right) \geq g\left(f^{-1}\left(\sum_{j=1}^{n} q_{j} f\left(\tilde{\gamma}_{\theta(j)}^{2}\right)\right)\right)$,

$f\left(g^{-1}\left(\sum_{j=1}^{n} q_{j} g\left(\eta_{\theta(j)}^{2}\right)\right)\right) \geq f\left(g^{-1}\left(\sum_{j=1}^{n} q_{j} g\left(\tilde{\eta}_{\theta(j)}^{2}\right)\right)\right)$,

Then it can be achieved that

$\frac{1}{n !} \sum_{\theta=1}^{n} g\left(f^{-1}\left(\sum_{j=1}^{n} q_{j} f\left(\gamma_{\theta(j)}^{2}\right)\right)\right) \geq$

$\frac{1}{n !} \sum_{\theta=1}^{n} g\left(f^{-1}\left(\sum_{j=1}^{n} q_{j} f\left(\tilde{\gamma}_{\theta(j)}^{2}\right)\right)\right)$,

$\frac{1}{n !} \sum_{\theta=1}^{n} f\left(g^{-1}\left(\sum_{j=1}^{n} q_{j} g\left(\eta_{\theta(j)}^{2}\right)\right)\right) \geq$

$\frac{1}{n !} \sum_{\theta=1}^{n} f\left(g^{-1}\left(\sum_{j=1}^{n} q_{j} g\left(\tilde{\eta}_{\theta(j)}^{2}\right)\right)\right)$.

Then it is obtained that

$g^{-1}\left(\frac{1}{n !} \sum_{\theta=1}^{n} g\left(f^{-1}\left(\sum_{j=1}^{n} q_{j} f\left(\gamma_{\theta(j)}^{2}\right)\right)\right)\right)$

$\geq g^{-1}\left(\frac{1}{n !} \sum_{\theta=1}^{n} g\left(f^{-1}\left(\sum_{j=1}^{n} q_{j} f\left(\tilde{\gamma}_{\theta(j)}^{2}\right)\right)\right)\right)$,

$f^{-1}\left(\frac{1}{n !} \sum_{\theta=1}^{n} f\left(g^{-1}\left(\sum_{j=1}^{n} q_{j} g\left(\eta_{\theta(j)}^{2}\right)\right)\right)\right)$

$\leq f^{-1}\left(\frac{1}{n !} \sum_{\theta=1}^{n} f\left(g^{-1}\left(\sum_{j=1}^{n} q_{j} g\left(\tilde{\eta}_{\theta(j)}^{2}\right)\right)\right)\right.$.

Then it can be achieved that

$\lambda f\left(g^{-1}\left(\frac{1}{n !} \sum_{\theta=1}^{n} g\left(f^{-1}\left(\sum_{j=1}^{n} q_{j} f\left(\gamma_{\theta(j)}^{2}\right)\right)\right)\right)\right)$

$\leq \lambda f\left(g^{-1}\left(\frac{1}{n !} \sum_{\theta=1}^{n} g\left(f^{-1}\left(\sum_{j=1}^{n} q_{j} f\left(\tilde{\gamma}_{\theta(j)}^{2}\right)\right)\right)\right)\right)$,

$\lambda g\left(f^{-1}\left(\frac{1}{n !} \sum_{\theta=1}^{n} f\left(g^{-1}\left(\sum_{j=1}^{n} q_{j} g\left(\eta_{\theta(j)}^{2}\right)\right)\right)\right)\right)$

$\leq \lambda g\left(f^{-1}\left(\frac{1}{n !} \sum_{\theta=1}^{n} f\left(g^{-1}\left(\sum_{j=1}^{n} q_{j} g\left(\tilde{\eta}_{\theta(j)}^{2}\right)\right)\right)\right)\right.$.

Then it is obtained that 


$$
\begin{aligned}
& f^{-1}\left(\lambda f\left(g^{-1}\left(\frac{1}{n !} \sum_{\theta=1}^{n} g\left(f^{-1}\left(\sum_{j=1}^{n} q_{j} f\left(\gamma_{\theta(j)}^{2}\right)\right)\right)\right)\right)\right) \\
& \geq f^{-1}\left(\lambda f\left(g^{-1}\left(\frac{1}{n !} \sum_{\theta=1}^{n} g\left(f^{-1}\left(\sum_{j=1}^{n} q_{j} f\left(\tilde{\gamma}_{\theta(j)}^{2}\right)\right)\right)\right)\right)\right), \\
& g^{-1}\left(\lambda g\left(f^{-1}\left(\frac{1}{n !} \sum_{\theta=1}^{n} f\left(g^{-1}\left(\sum_{j=1}^{n} q_{j} g\left(\eta_{\theta(j)}^{2}\right)\right)\right)\right)\right)\right) \\
& \leq g^{-1}\left(\lambda g\left(f^{-1}\left(\frac{1}{n !} \sum_{\theta=1}^{n} f\left(g^{-1}\left(\sum_{j=1}^{n} q_{j} g\left(\tilde{\eta}_{\theta(j)}^{2}\right)\right)\right)\right)\right)\right)
\end{aligned}
$$

Then it can be achieved that

$$
\begin{aligned}
& \sqrt{f^{-1}\left(\lambda f\left(g^{-1}\left(\frac{1}{n !} \sum_{\theta=1}^{n} g\left(f^{-1}\left(\sum_{j=1}^{n} q_{j} f\left(\gamma_{\theta(j)}^{2}\right)\right)\right)\right)\right)\right.} \\
& \geq \sqrt{f^{-1}\left(\lambda f\left(g^{-1}\left(\frac{1}{n !} \sum_{\theta=1}^{n} g\left(f^{-1}\left(\sum_{j=1}^{n} q_{j} f\left(\tilde{\gamma}_{\theta(j)}^{2}\right)\right)\right)\right)\right)\right.}, \\
& \sqrt{g^{-1}\left(\lambda g\left(f^{-1}\left(\frac{1}{n !} \sum_{\theta=1}^{n} f\left(g^{-1}\left(\sum_{j=1}^{n} q_{j} g\left(\eta_{\theta(j)}^{2}\right)\right)\right)\right)\right)\right)} \\
& \leq \sqrt{g^{-1}\left(\lambda g\left(f^{-1}\left(\frac{1}{n !} \sum_{\theta=1}^{n} f\left(g^{-1}\left(\sum_{j=1}^{n} q_{j} g\left(\tilde{\eta}_{\theta(j)}^{2}\right)\right)\right)\right)\right)\right.},
\end{aligned}
$$

Since

$\left.\operatorname{S(PHFAMM}{ }^{Q}\left(h_{1 p}, h_{2 p}, \ldots h_{n p}\right)\right)$

$=\frac{1}{2}\left(1+\frac{1}{l_{h}} \sum_{(\gamma, \eta) \in(\mu, v)}\left[F_{1}-G_{1}\right]\right)$,

where

$$
\begin{aligned}
& F_{1}=f^{-1}\left(\lambda f\left(g^{-1}\left(\frac{1}{n !} \sum_{\theta=1}^{n} g\left(f^{-1}\left(\sum_{j=1}^{n} q_{j} f\left(\gamma_{\theta(j)}^{2}\right)\right)\right)\right)\right)\right), \\
& G_{1}=g^{-1}\left(\lambda g\left(f^{-1}\left(\frac{1}{n !} \sum_{\theta=1}^{n} f\left(g^{-1}\left(\sum_{j=1}^{n} q_{j} g\left(\eta_{\theta(j)}^{2}\right)\right)\right)\right)\right)\right) . \\
& S\left(\operatorname{PHFAMM}^{Q}\left(\tilde{h}_{1 p}, \tilde{h}_{2 p}, \ldots \tilde{h}_{n p}\right)\right)= \\
& \frac{1}{2}\left(1+\frac{1}{l_{h}} \sum_{(\gamma, \eta) \in(\mu, v)}\left[\tilde{F}_{1}-\tilde{G}_{1}\right]\right),
\end{aligned}
$$

where

$$
\begin{aligned}
& \tilde{F}_{1}=f^{-1}\left(\lambda f\left(g^{-1}\left(\frac{1}{n !} \sum_{\theta=1}^{n} g\left(f^{-1}\left(\sum_{j=1}^{n} q_{j} f\left(\tilde{\gamma}_{\theta(j)}^{2}\right)\right)\right)\right)\right),\right. \\
& \tilde{G}_{1}=g^{-1}\left(\lambda g\left(f^{-1}\left(\frac{1}{n !} \sum_{\theta=1}^{n} f\left(g^{-1}\left(\sum_{j=1}^{n} q_{j} g\left(\tilde{\eta}_{\theta(j)}^{2}\right)\right)\right)\right)\right)\right) .
\end{aligned}
$$

Therefore, it is obtained that

$\left.\operatorname{SPHFAMM}^{Q}\left(h_{1 p}, h_{2 p}, \ldots h_{n p}\right)\right) \geq S\left(\operatorname{PHFAMM}^{Q}\left(\tilde{h}_{1 p}, \tilde{h}_{2 p}, \ldots \tilde{h}_{n p}\right)\right)$. , and $\operatorname{PHFAMM}^{Q}\left(h_{1 p}, h_{2 p}, \ldots h_{n p}\right) \geq \operatorname{PHFAMM}^{Q}\left(\tilde{h}_{1 p}, \tilde{h}_{2 p}, \ldots \tilde{h}_{n p}\right)$ holds.

Theorem 4(Boundness). Let

$h_{j p}=\bigcup_{\substack{\left(\gamma_{j}, \eta_{j}\right) \in\left(\mu_{j}, v_{j}\right) \\ n=1,2, \ldots n}}\left\{\left(\gamma_{j}, \eta_{j}\right)\right\}$ be a collection of PHF-

Ns, and let $\gamma_{\min }=\min \left\{\gamma_{j_{\min }}\right\}$,

where $\gamma_{j_{\min }}=\min _{\left(\gamma_{j}, \eta_{j}\right) \in\left(\mu_{j}, v_{j}\right)}\left\{\gamma_{j}\right\}$ for all $i=1,2, \ldots n$;

$\gamma_{\max }=\max \left\{\gamma_{j_{\max }}\right\}$,

where $\gamma_{j_{\max }}=\max _{\left(\gamma_{j}, \eta_{j}\right) \in\left(\mu_{j}, v_{j}\right)}\left\{\gamma_{j}\right\}$ for all $i=1,2, \ldots n$; $\eta_{\text {min }}=\min \left\{\eta_{j_{\text {min }}}\right\}$

where $\eta_{j_{\min }}=\min _{\left(\gamma_{j}, \eta_{j}\right) \in\left(\mu_{j}, v_{j}\right)}\left\{\eta_{j}\right\}$ for all $i=1,2, \ldots n$;

$\eta_{\text {max }}=\max \left\{\eta_{j_{\max }}\right\}$,

where $\eta_{j_{\max }}=\max _{\left(\gamma_{j}, \eta_{j}\right) \in\left(\mu_{j}, v_{j}\right)}\left\{\eta_{j}\right\}$ for all $i=1,2, \ldots n$.

Let $h_{p-}=\left(\gamma_{\min }, \eta_{\max }\right)$ and $h_{p+}=\left(\gamma_{\max }, \eta_{\min }\right)$, then

$$
h_{p-} \leq \operatorname{PHFAMM}^{Q}\left(h_{1 p}, h_{2 p}, \ldots h_{n p}\right) \leq h_{p+} .
$$

Proof.

According to Theorem 2 and Theorem 3, it can be achieved that

$\operatorname{PHFAMM}^{Q}\left(h_{1 p}, h_{2 p}, \ldots h_{n p}\right) \leq \operatorname{PHFAMM}^{Q}\left(h_{p+}, h_{p+}, \ldots h_{p+}\right)$,

$\operatorname{PHFAMM}^{Q}\left(h_{1 p}, h_{2 p}, \ldots h_{n p}\right) \geq \operatorname{PHFAMM}^{Q}\left(h_{p-}, h_{p-}, \ldots h_{p-}\right)$.

Consequently, it is obtained that

$$
h_{p-} \leq \operatorname{PHFAMM}^{Q}\left(h_{1 p}, h_{2 p}, \ldots h_{n p}\right) \leq h_{p+} .
$$

Equation (13) is a generalized expression of the PHFAMM operator. To construct specific expressions of the operator, different pairs of $f(x)_{s}$ and $g(x)_{s}$ are used:

- (Algebraic) If $f(x)=-\ln (t)$, then the PHFAMM operator reduces to a Pythagorean hesitant fuzzy Archimedean Algebraic Muirhead mean (PHFAAMM) operator:

$$
\begin{aligned}
& \operatorname{PHFAAMM}^{Q}\left(h_{1 p}, h_{2 p}, \ldots h_{n p}\right) \\
& =\bigcup_{\substack{\left(\gamma_{j}, \eta_{j}\right) \in\left(\mu_{j}, v_{j}\right) \\
i=1,2 \ldots n}}\left\{\left(\sqrt{\left(1-\mu_{A}\right)^{\lambda}}, \sqrt{1-\left(1-v_{A}\right)^{\lambda}}\right)\right\}
\end{aligned}
$$

where

$$
\mu_{A}=\prod_{\theta \in S_{n}}\left(1-\prod_{j=1}^{n} \gamma_{\theta(j)}^{2 q_{j}}\right)^{\frac{1}{n !}}, v_{A}=\prod_{\theta \in S_{n}}\left(1-\prod_{j=1}^{n}\left(1-\eta_{\theta(j)}^{2}\right)^{q_{j}}\right)^{\frac{1}{n !}}
$$

- (Einstein) If $f(x)=\ln \left(\frac{2-t}{t}\right)$, then the PHFAMM operator reduces to a Pythagorean Hesitant Fuzzy Archimedean Einstein Muirhead mean (PHFAEMM) operator:

$$
\begin{aligned}
& \operatorname{PHFAEMM}^{Q}\left(h_{1 p}, h_{2 p}, \ldots h_{n p}\right) \\
& =\bigcup_{\substack{\left(\gamma_{j}, \eta_{j}\right) \in\left(\mu_{j}, v_{j}\right) \\
j=1,2 \ldots n}} \sqrt{\frac{2\left(u_{1}-u_{2}\right)^{\lambda}}{\left(\frac{\left(v_{1}+3 v_{2}\right)^{\lambda}-\left(v_{1}-v_{2}\right)^{\lambda}}{\left(v_{1}+3 v_{2}\right)^{\lambda}+\left(v_{1}-v_{2}\right)^{\lambda}}\right)}},
\end{aligned}
$$

where

$$
\begin{aligned}
& u_{00}=\prod_{j=1}^{n}\left(2-\gamma_{\theta(j)}^{2}\right)^{q_{j}}, u_{01}=\prod_{j=1}^{n} \gamma_{\theta(j)}^{2 q_{j}}, \\
& u_{1}=\prod_{\theta \in S_{n}}\left(u_{00}+3 u_{01}\right)^{\frac{1}{n !}}, u_{2}=\prod_{\theta \in S_{n}}\left(u_{00}-u_{01}\right)^{\frac{1}{n !}}, \\
& v_{00}=\prod_{j=1}^{n}\left(1+\eta_{\theta(j)}^{2}\right)^{q_{j}}, v_{01}=\prod_{j=1}^{n}\left(1-\eta_{\theta(j)}^{2}\right)^{q_{j}},
\end{aligned}
$$




$$
v_{1}=\prod_{\theta \in S_{n}}\left(v_{00}+3 v_{01}\right)^{\frac{1}{n !}}, v_{2}=\prod_{\theta \in S_{n}}\left(v_{00}-v_{01}\right)^{\frac{1}{n !}} .
$$

- (Hamacher) If $f(x)=\ln \left(\frac{\theta+(1-\theta) t}{t}\right), \theta>0, \delta=$ $\theta-1$, then the PHFAMM operator reduces to a Pythagorean hesitant fuzzy Archimedean Hamacher Muirhead mean (PHFAHMM) operator:

$$
\begin{aligned}
& \operatorname{PHFAHMM}^{Q}\left(h_{1 p}, h_{2 p}, \ldots h_{n p}\right) \\
& =\bigcup_{\substack{\left(\gamma_{j}, \eta_{j}\right) \in\left(\mu_{j}, v_{j}\right) \\
i=1,2 \ldots n}}\left\{\left(\sqrt{\frac{\theta U_{2}}{U_{1}+\delta U_{2}}}, \sqrt{\frac{V_{1}-V_{2}}{V_{1}+\delta V_{2}}}\right)\right\}
\end{aligned}
$$

where

$$
\begin{aligned}
& U_{1}=\left(u_{1}+\left(\theta^{2}-1\right) u_{2}\right)^{\lambda}, U_{2}=\left(u_{1}-u_{2}\right)^{\lambda}, \\
& V_{1}=\left(\left(\theta^{2}-1\right) v_{1}+v_{2}\right)^{\lambda}, V_{2}=\left(v_{2}-v_{1}\right)^{\lambda}, \\
& \left.u_{00}=\prod_{j=1}^{n}(\theta-\delta) \gamma_{\theta(j)}^{2}\right)^{q_{j}}, u_{01}=\prod_{j=1}^{n} \gamma_{j \theta(j)}^{2 q_{j}}, \\
& u_{1}=\prod_{\vartheta \in S_{n}}\left(u_{00}+\left(\theta^{2}-1\right) u_{01}\right)^{\frac{1}{n !}}, u_{2}=\prod_{\vartheta \in S_{n}}\left(u_{00}-u_{01}\right)^{\frac{1}{n !},} \\
& v_{00}=\prod_{j=1}^{n}\left(\theta-\delta\left(1-\eta_{\theta(j)}^{2}\right)\right)^{q_{j}}, v_{01}=\prod_{j=1}^{n}\left(1-\eta_{\theta(j)}^{2}\right)^{q_{j}}, \\
& v_{1}=\prod_{\vartheta \in S_{n}}\left(v_{00}-v_{01}\right)^{\frac{1}{n !}}, v_{2}=\prod_{\vartheta \in S_{n}}\left(v_{00}+\left(\theta^{2}-1\right) v_{01}\right)^{\frac{1}{n !} .}
\end{aligned}
$$

The PHFAHMM operator reduces to the PHFAAMM operator when $\theta=1$ and reduces to the PHFAEMM operator when $\theta=2$.

- (Frank) If $f(x)=\ln \left(\frac{\theta-1}{\theta^{t}-1}\right), \theta>1, \delta=\theta-1$, then the PHFAMM operator reduces to a Pythagorean hesitant fuzzy Archimedean Frank Muirhead mean (PHFAFMM) operator:

$$
\begin{aligned}
& \operatorname{PHFAFMM}^{Q}\left(h_{1 p}, h_{2 p}, \ldots h_{n p}\right) \\
& =\bigcup_{\substack{\left(\gamma_{j}, \eta_{j}\right) \in\left(\mu_{j}, v_{j}\right) \\
i=1,2 \ldots n}}\left\{\sqrt{\sqrt{\log _{\theta}^{\frac{\delta U_{0}+U_{1}}{U_{2}}}}},\right. \\
& \left.\left.\sqrt{1-\log _{\theta}^{\frac{\delta V_{0}+V_{1}}{V_{2}}}}\right)\right\}
\end{aligned}
$$

where

$$
\begin{aligned}
& U_{0}=\left(\delta\left(u_{1}-u_{2}\right)\right)^{\lambda}, U_{1}=\left(\delta^{2} u_{2}+\delta u_{1}\right)^{\lambda}, \\
& U_{2}=\left(\delta^{2} u_{2}+\delta u_{1}\right)^{\lambda}, \\
& V_{0}=\left(\delta\left(v_{1}-v_{2}\right)\right)^{\lambda}, V_{1}=\left(\delta^{2} v_{2}+\delta v_{1}\right)^{\lambda}, \\
& V_{2}=\left(\delta^{2} v_{2}+\delta v_{1}\right)^{\lambda}, \\
& u_{00}=\prod_{j=1}^{n}\left(\theta^{\gamma^{2}(j)}-1\right)^{q_{j}}, u_{01}=\prod_{j=1}^{n} \delta^{q_{j}}, \\
& u_{1}=\prod_{9 \in S_{n}}\left(\delta^{2} u_{00}+\delta u_{01}\right)^{\frac{1}{n !}},
\end{aligned}
$$

$$
\begin{aligned}
& u_{2}=\prod_{\vartheta \in S_{n}}\left(\delta\left(u_{01}-u_{00}\right)\right)^{\frac{1}{n !}}, \\
& v_{00}=\prod_{j=1}^{n}\left(\theta^{1-\eta_{\theta(j)}^{2}}-1\right)^{q_{j}}, v_{01}=\prod_{j=1}^{n} \delta^{q_{j}}, \\
& v_{1}=\prod_{\vartheta \in S_{n}}\left(\delta^{2} v_{00}+\delta v_{01}\right)^{\frac{1}{n !}}, v_{2}=\prod_{\vartheta \in S_{n}}\left(\delta\left(v_{01}-v_{00}\right)\right)^{\frac{1}{n !}} .
\end{aligned}
$$

\subsection{WPHFAMM Operator}

The PHFAMM operator has advantages in describing fuzzy information flexibly and capturing the interrelationships among criteria. But it does not measure the relative importance of criteria. To this end, weights are introduced and a weighted PHFAMM operator is constructed as follow:

Definition 12. On the basis of Definition 7, let $w=$ $\left(w_{1}, w_{2}, \ldots, w_{n}\right)^{T}$ be the weight vector of $h_{j p}$ (where $w_{j}$ delegates the importance degree of $h_{j p}$ ) such that $w_{j} \in[0,1]$ and $\sum_{j=1}^{n} w_{j}=1$. If

$$
\begin{aligned}
& \operatorname{WPHFAMM}^{Q}\left(h_{1 p}, h_{2 p}, \ldots h_{n p}\right) \\
& =\left(\frac{1}{n !}\left(\bigoplus_{\vartheta \in S_{n}} \bigotimes_{j=1}^{n}\left(n w_{p \vartheta_{(j)}} h_{p \vartheta_{(j)}}\right) q^{q_{j}}\right)\right)^{\frac{1}{\sum_{j=1}^{n} q_{j}}}
\end{aligned}
$$

Then WPHFAMM ${ }^{\mathrm{Q}}$ is called the WPHFAMM operator.

Theorem 5. Let $h_{j p}=\left(u_{j}, v_{j}\right)(j=1,2, \ldots n)$ be a set of PHFNs, and $Q=\left(q_{1}, q_{2}, \ldots, q_{n}\right) \in R^{n}$ be a parameter vector. Then WPHFAMM ${ }^{Q}\left(h_{1 p}, h_{2 p}, \ldots h_{n p}\right)$ is still a PHFN, and

$$
\begin{aligned}
& \operatorname{WPHFAMM}^{Q}\left(h_{1 p}, h_{2 p}, \ldots h_{n p}\right) \\
& =\bigcup_{\substack{\left.\gamma_{j}, \eta_{j}\right) \in\left(\mu_{j}, v_{j}\right) \\
j=1, \ldots, n}}\left\{\sqrt{f^{-1}\left(\lambda f\left(g^{-1}\left(\frac{1}{n !} \cdot u\right)\right)\right)},\right. \\
& \left.\left.\sqrt{g^{-1}\left(\lambda g\left(f^{-1}\left(\frac{1}{n !} \cdot v\right)\right)\right)}\right)\right\}
\end{aligned}
$$

where, $\lambda=\frac{1}{\sum_{j=1}^{n} q_{j}}$,

$$
\begin{aligned}
& u=\sum_{\theta=1}^{n} g\left(f^{-1}\left(\sum_{j=1}^{n} q_{j} f\left(g^{-1}\left(n w_{j} g\left(\gamma_{\theta(j)}^{2}\right)\right)\right)\right)\right), \\
& v=\sum_{\theta=1}^{n} f\left(g^{-1}\left(\sum_{j=1}^{n} q_{j} g\left(f^{-1}\left(n w_{j} f\left(\eta_{\theta(j)}^{2}\right)\right)\right)\right)\right) .
\end{aligned}
$$

The proof of Theorem 5 is similar as that of Theorem 1 and is omitted here. Furthermore, the WPHFAMM operator has the properties of monotonicity and boundedness. Such proofs are respectively similar as that of Theorem 3 and Theorem 4 and are also omitted here.

The following are some special cases of the WPHFAMM operator with respect to the value of parameter $Q$ : 
(1) When $Q=(1,0, \ldots, 0)$, the WPHFAMM reduces to:

$$
\begin{aligned}
& \operatorname{WPHFAMM}^{Q}\left(h_{1 p}, h_{2 p}, \ldots h_{n p}\right) \\
& =\bigcup_{\substack{\left(\gamma_{j}, \eta_{j}\right) \in\left(\mu_{j}, v_{j}\right) \\
j=1,2, \ldots n}}\left\{\left(\sqrt{g^{-1}\left(\sum_{i=1}^{n} w_{j} g\left(\gamma_{\theta(j)}^{2}\right)\right.}\right),\right.
\end{aligned}
$$

which is the APHFWA operator presented in [35].

(2) When $Q=\left(\frac{1}{n}, \frac{1}{n}, \ldots, \frac{1}{n}\right)$, the WPHFAMM reduces to:

$$
\begin{aligned}
& \operatorname{WPHFAMM}^{Q}\left(h_{1 p}, h_{2 p}, \ldots h_{n p}\right) \\
& =\bigcup_{\substack{\left(\gamma_{j}, \eta_{j}\right) \in\left(\mu_{j}, v_{j}\right) \\
j=1,2, \ldots, n}}\left\{\sqrt{f^{-1}\left(\sum_{j=1}^{n} \frac{1}{n} f\left(\gamma_{\theta(j)}^{2}\right)\right)},\right.
\end{aligned}
$$

which is the APHFWG operator presented in [35].

(3) When $\mathrm{Q}=(1,1,0,0, \ldots, 0)$, the WPHFAMM reduces to:

$$
\begin{aligned}
& \operatorname{WPHFAMM}^{Q}\left(h_{1 p}, h_{2 p}, \ldots h_{n p}\right) \\
& \left.=\bigcup_{\substack{\left(\gamma_{i, j}, \eta_{i, j}\right) \in\left(\mu_{i, j}, v_{i, j}\right) \\
i, j=1,2, \ldots, n, i \neq j}}\left\{\sqrt{f^{-1}\left(\frac{U_{B M}}{p+q}\right)}, \sqrt{g^{-1}\left(\frac{V_{B M}}{p+q}\right)}\right)\right\}
\end{aligned}
$$

where $p, q \geq 0$, and

$U_{B M}=f\left(g^{-1}\left(\frac{1}{n(n-1)} \sum_{i, j=1, i \neq j}^{n} g\left(f^{-1}\left(p f\left(\gamma_{i}^{2}\right)+q f\left(\gamma_{j}^{2}\right)\right)\right)\right)\right)$, $V_{B M}=g\left(f^{-1}\left(\frac{1}{n(n-1)} \sum_{i, j=1, i \neq j}^{n} f\left(g^{-1}\left(p g\left(\eta_{i}^{2}\right)+q g\left(\eta_{j}^{2}\right)\right)\right)\right)\right)$.

(4) When $Q=(\underbrace{1,1, \ldots 1}_{k}, \underbrace{0,0, \ldots 0}_{n-k})$, the WPHFAMM reduces to:

$$
\begin{aligned}
& \operatorname{WPHFAMM}^{Q}\left(h_{1 p}, h_{2 p}, \ldots h_{n p}\right) \\
& =\bigcup_{\substack{\left(\gamma_{i j}, \eta_{i, j} \in \in\left(\mu_{i,}, v_{i, j}\right) \\
i=1,1, \ldots, j, 1,2, \ldots k\right.}}\left\{\left(\sqrt{f^{-1}\left(U_{M S M}\right)}, \sqrt{g^{-1}\left(V_{M S M}\right)}\right)\right\}
\end{aligned}
$$

whose Algebraic form is the WHPFMSM operator presented in [31], where

$U_{M S M}=\frac{1}{k} f\left(g^{-1}\left(\frac{1}{C_{n}^{k}} \sum_{1 \leq i_{1}<\ldots<i_{k} \leq n} g\left(f^{-1}\left(\sum_{j=1}^{k} w_{i_{j}} f\left(\gamma_{i_{j}}^{2}\right)\right)\right)\right)\right)$,

$V_{M S M}=\frac{1}{k} g\left(f^{-1}\left(\frac{1}{C_{n}^{k}} \sum_{1 \leq i_{1}<\ldots<i_{k} \leq n} f\left(g^{-1}\left(\sum_{j=1}^{k} w_{i_{j}} g\left(\eta_{i_{j}}^{2}\right)\right)\right)\right)\right)$.

Equation (19) is a generalized expression of the WPHFAMM operator. To construct specific expressions of the operator, different pairs of $f(x)_{s}$ and $g(x)_{s}$ are used:

- (Algebraic) If $f(x)=-\ln (t)$, the WPHFAMM operator reduces to a weighted PHFAAMM operator (WPHFAAMM):

$$
\begin{aligned}
& \operatorname{WPHFAAMM}^{Q}\left(h_{1 p}, h_{2 p}, \ldots h_{n p}\right) \\
& =\bigcup_{\substack{\left(\gamma_{j}, \eta_{j}\right) \in\left(\mu_{j}, v_{j}\right) \\
j=1,2 \ldots n}}\left\{\left(\sqrt{\left(1-u_{W A}\right)^{\lambda}}, \sqrt{1-\left(1-v_{W A}\right)^{\lambda}}\right)\right\}
\end{aligned}
$$

where

$$
\begin{aligned}
& u_{W A}=\prod_{\theta \in S_{n}}\left(1-\prod_{j=1}^{n}\left(1-\left(1-\gamma_{\theta(j)}^{2}\right)^{n w_{j}}\right)^{q_{j}}\right)^{\frac{1}{n !}}, \\
& v_{W A}=\prod_{\theta \in S_{n}}\left(1-\prod_{j=1}^{n}\left(1-\eta_{\theta(j)}^{2 n w_{j}}\right)^{q_{j}}\right)^{\frac{1}{n !}} .
\end{aligned}
$$

- (Einstein) If $f(x)=\ln \left(\frac{2-t}{t}\right)$, then the WPHFAMM operator reduces to a weighted PHFAEMM operator (WPHFAEMM):

$$
\begin{aligned}
& \text { WPHFAEMM }^{Q}\left(h_{1 p}, h_{2 p}, \ldots h_{n p}\right) \\
& =\bigcup_{\substack{\left(\gamma_{j}, \eta_{j}\right) \in\left(\mu_{j}, v_{j}\right) \\
j=1,2 \ldots n}}\left\{\sqrt{\frac{2\left(u_{1}-u_{2}\right)^{\lambda}}{\left(\frac{\left(u_{1}+3 u_{2}\right)^{\lambda}+\left(u_{1}-u_{2}\right)^{\lambda}}{\left(v_{1}+3 v_{2}\right)^{\lambda}-\left(v_{1}-v_{2}\right)^{\lambda}+\left(v_{1}-v_{2}\right)^{\lambda}}\right.}},\right\}
\end{aligned}
$$

where

$$
\begin{aligned}
& u_{00}=\prod_{j=1}^{n}\left(\left(1+\gamma_{\theta(j)}^{2}\right)^{n w_{j}}+3\left(1-\gamma_{\theta(j)}^{2}\right)^{n w_{j}}\right)^{q_{j}}, \\
& u_{01}=\prod_{j=1}^{n}\left(\left(1+\gamma_{\theta(j)}^{2}\right)^{n w_{j}}-\left(1-\gamma_{\theta(j)}^{2}\right)^{n w_{j}}\right)^{q_{j}}, \\
& u_{1}=\prod_{\vartheta \in S_{n}}\left(u_{00}+3 u_{01}\right)^{\frac{1}{n !}}, u_{2}=\prod_{\vartheta \in S_{n}}\left(u_{00}-u_{01}\right)^{\frac{1}{n !}}, \\
& v_{00}=\prod_{j=1}^{n}\left(\left(2-\eta_{\theta(j)}^{2}\right)^{n w_{j}}+3 \eta_{\theta(j)}^{2 n w_{j}}\right)^{q_{j}}, \\
& v_{01}=\prod_{j=1}^{n}\left(\left(2-\eta_{\theta(j)}^{2}\right)^{n w_{j}}-\eta_{\theta(j)}^{2 n w_{j} 2}\right)^{q_{j}}, \\
& v_{1}=\prod_{\vartheta \in S_{n}}\left(v_{00}+3 v_{01}\right)^{\frac{1}{n !}}, v_{2}=\prod_{\vartheta \in S_{n}}\left(v_{00}-v_{01}\right)^{\frac{1}{n !} .}
\end{aligned}
$$

- (Hamacher) If $f(x)=\ln \left(\frac{\theta+(1-\theta) t}{t}\right), \theta>0, \delta=$ $\theta-1$, the WPHFAMM operator reduces to a weighted PHFAHMM operator (WPHFAHMM):

$$
\begin{aligned}
& \text { WPHFAHMM }^{Q}\left(h_{1 p}, h_{2 p}, \ldots h_{n p}\right) \\
& =\bigcup_{\substack{\left(y_{j}, n_{i}\right) \in\left(\mu_{j}, v_{j}\right) \\
j=1,2 \ldots n}} \sqrt\left[\left\{\left(\sqrt{\frac{\theta\left(u_{1}-u_{2}\right)^{\lambda}}{U_{1}+\delta\left(u_{1}-u_{2}\right)^{\lambda}}},\right]{\left.\left.\sqrt{\frac{V_{1}-\left(v_{1}-v_{2}\right)^{\lambda}}{V_{1}+\delta\left(v_{1}-v_{2}\right)^{\lambda}}}\right)\right\}}\right.\right.
\end{aligned}
$$

where

$$
\begin{aligned}
& U_{1}=\left(u_{1}+\left(\theta^{2}-1\right) u_{2}\right)^{\lambda}, V_{1}=\left(v_{1}+\left(\theta^{2}-1\right) v_{2}\right)^{\lambda}, \\
& u_{00}=\prod_{j=1}^{n}\left(\left(\theta-\delta\left(1-\gamma_{\theta(j)}^{2}\right)\right)^{n w_{j}}+\left(\theta^{2}-1\right)\left(1-\gamma_{\theta(j)}^{2}\right)^{n w_{j}}\right)^{q_{j}}, \\
& u_{01}=\prod_{j=1}^{n}\left(\left(\theta-\delta\left(1-\gamma_{\theta(j)}^{2}\right)\right)^{n w_{j}}-\left(1-\gamma_{\theta(j)}^{2}\right)^{n w_{j}}\right)^{q_{j}},
\end{aligned}
$$




$$
\begin{aligned}
& u_{1}=\prod_{\vartheta \in S_{n}}\left(u_{00}+\left(\theta^{2}-1\right) u_{01}\right)^{\frac{1}{n !}}, u_{2}=\prod_{\vartheta \in S_{n}}\left(u_{00}-u_{01}\right)^{\frac{1}{n !}}, \\
& v_{00}=\prod_{j=1}^{n}\left(\left(\theta-\delta \eta_{\theta(j)}^{2}\right)^{n w_{j}}+\left(\theta^{2}-1\right) \eta_{\theta(j)}^{2 n w_{j}}\right)^{q_{j}}, \\
& v_{01}=\prod_{j=1}^{n}\left(\left(\theta-\delta \eta_{\theta(j)}^{2}\right)^{n w_{j}}-\eta_{\theta(j)}^{2 n w_{j}}\right)^{q_{j}}, \\
& v_{1}=\prod_{\vartheta \in S_{n}}\left(v_{00}+\left(\theta^{2}-1\right) v_{01}\right)^{\frac{1}{n !}}, v_{2}=\prod_{\vartheta \in S_{n}}\left(v_{00}-v_{01}\right)^{\frac{1}{n !}} .
\end{aligned}
$$

The WPHFAHMM operator reduces to the WPHFAAMM operator when $\theta=1$ and reduces to the WPHFAEMM operator when $\theta=2$.

- (Frank) If $f(x)=\ln \left(\frac{\theta-1}{\theta^{t}-1}\right), \theta>1, \delta=\theta-1$, the WPHFAMM operator reduces to a weighted PHFAFMM operator (WPHFAFMM):

$$
\begin{aligned}
& \text { WPHFAFMM } \\
& =\bigcup_{\substack{\left(\gamma_{j}, \eta_{j}\right) \in\left(\mu_{j}, v_{j}\right) \\
j=1,2 \ldots n}}\left\{\sqrt{\sqrt{\log _{\theta} \frac{\delta\left(\delta\left(u_{2}-u_{1}\right)\right)^{\lambda}+\left(\delta^{2} u_{1}+\delta u_{2}\right)^{\lambda}}{\left(\delta^{2} u_{1}+\delta u_{2}\right)^{\lambda}}}},\right. \\
& \left.\left.\log _{\theta}^{\frac{\delta\left(\delta\left(v_{2}-v_{1}\right)\right)^{\lambda}+\left(\delta^{2} v_{1}+\delta v_{2}\right)^{\lambda}}{\left(\delta^{2} v_{1}+\delta v_{2}\right)^{\lambda}}}\right)\right\}
\end{aligned}
$$

where

$$
\begin{aligned}
& u_{00}=\prod_{j=1}^{n}\left(\delta^{2}\left(\theta^{1-\gamma_{\theta(j)}^{2}}-1\right)^{n w_{j}}+\delta^{n w_{j}+1}\right)^{q_{j}}, \\
& u_{01}=\prod_{j=1}^{n}\left(\delta^{n w_{j}+1}-\delta\left(\theta^{1-\gamma_{\theta(j)}^{2}}-1\right)^{n w_{j}}\right)^{q_{j}}, \\
& u_{1}=\prod_{\vartheta \in S_{n}}\left(\delta\left(u_{00}-u_{01}\right)\right)^{\frac{1}{n !}}, u_{2}=\prod_{\vartheta \in S_{n}}\left(\delta^{2} u_{01}+\delta u_{00}\right)^{\frac{1}{n !}}, \\
& v_{00}=\prod_{j=1}^{n}\left(\delta^{2}\left(\theta^{\eta_{\theta(j)}^{2}}-1\right)^{n w_{j}}+\delta^{n w_{j}+1}\right)^{q_{j}}, \\
& v_{01}=\prod_{j=1}^{n}\left(\delta^{n w_{j}+1}-\delta\left(\theta^{\eta_{\theta(j)}^{2}}-1\right)^{n w_{j}}\right)^{q_{j}}, \\
& v_{1}=\prod_{\vartheta \in S_{n}}\left(\delta\left(v_{00}-v_{01}\right)\right)^{\frac{1}{n !}}, v_{2}=\prod_{\vartheta \in S_{n}}\left(\delta^{2} v_{01}+\delta v_{00}\right)^{\frac{1}{n !} .}
\end{aligned}
$$

\section{MCDM Method}

Let $\mathrm{Z}=\left\{z_{1}, z_{2}, \ldots, z_{m}\right\}$ be a set of alternatives, $\mathrm{C}=$ $\left\{c_{1}, c_{2}, \ldots, c_{n}\right\}$ be a collection of criteria whose weight vector is given by $w=\left(w_{1}, w_{2}, \ldots, w_{n}\right)^{T}$, with $w_{j} \in$ $[0,1]$ and $\sum_{j=1}^{n} w_{j}=1$. Suppose $H=\left(h_{p \vartheta_{(j)}}\right)_{m \times n}$ is a Pythagorean hesitant fuzzy decision matrix. Then, a MCDM method based on the PHFAMM and WPHFAMM operators can described via the following steps:

Step 1. Normalize the Pythagorean hesitant fuzzy decision matrix. Normally, there are two kinds of criteria in MCDM, i.e. benefit criteria and cost criteria. They respectively have a positive and negative impact on decision making. To remove the negative impact, the matrix needs to be normalized by

$$
h_{p \vartheta_{(j)}}=\left\{\begin{array}{l}
\left(\mu_{i p \vartheta_{(j)}}, v_{i p \vartheta_{(j)}}\right), \text { for benefit critera } \\
\left(v_{i p \vartheta_{(j)}}, \mu_{i p \vartheta_{(j)}}\right), \text { for cost critera }
\end{array}\right.
$$

Step 2. Calculate the comprehensive values of all alternatives. Taking $\left(h_{p \vartheta_{(j)}}\right)_{m \times n}, Q$ and $w$ as input, the comprehensive value for each alternative $z_{i}$ can be calculated using the PHFAMM (or WPHFAMM) operator.

$$
\begin{aligned}
h_{p} & =\operatorname{PHFAMM}^{Q}\left(h_{1 p}, h_{2 p}, \ldots h_{n p}\right) \\
\text { or } \quad h_{p} & =\operatorname{WPHFAMM}^{Q}\left(h_{1 p}, h_{2 p}, \ldots h_{n p}\right)
\end{aligned}
$$

Step 3. Calculate the scores and accuracies. According to Equation (6) and Equation (7), the scores and accuracies of the comprehensive values of all alternatives are calculated as

$$
\begin{aligned}
S\left(h_{p}\right) & =\frac{1}{2}\left(1+\frac{1}{l_{h}} \sum_{(\gamma, \eta) \in(\mu, v)}\left(\gamma^{2}-\eta^{2}\right)\right) \\
A\left(h_{p}\right) & =\frac{1}{l_{h}} \sum_{(\gamma, \eta) \in(\mu, \nu)}\left(\gamma^{2}-\eta^{2}\right)
\end{aligned}
$$

Step 4. Generate a sort of all alternatives. According to Definition 4 and the score and accuracy values of each alternative, a sort of all alternatives is generated.

\section{Example, Experiments and Comparisons}

In this section, an example is given to illustrate the working process of the proposed method firstly. Then a set of experiments are performed to explore the decision results under different parameters. Finally, the effectiveness of the method is demonstrated via comparisons with existing methods.

\subsection{Example}

With the fast-growing industrialization, the society is facing various threats, such as global warming, food safety, trash-dumping issue, natural resources depletion, etc. The green supply chain management (GSCM) (cited from Reference [35]) is one of burgeoning fields of research that present solutions for these threats. It not only considers environmental impacts but also productivity and profit. Consequently, GSCM is a good example of MCDM problems. It is formally described as follow.

There are five alternative green suppliers in GSCM $Z=\left\{z_{i} \mid i=1,2,3,4,5\right\}$. The experts mainly consider four criteria, namely, the product quality factor $\left(C_{1}\right)$, 
the environmental factor $\left(C_{2}\right)$, the delivery factor $\left(C_{3}\right)$, and the price factor $\left(C_{4}\right)$. The weight vector of these criteria is $w=(0.4,0.1,0.2,0.3)^{T}$. The assessed values of the four criteria of the five alternative green suppliers are shown in Table 3.

With the conditions above, the GSCM problem can be resolved using the proposed MCDM method. The specific process is as follows:

Step 1. Normalize the decision matrix. Since the four criteria are all benefit criteria, normalization is not needed.

Step 2. Calculate the comprehensive values of all alternatives. The comprehensive value $h_{p}$ for each alternative $z_{i}$ can be obtained via using the presented PHFAMM (or WPHFAMM) operator. Taking $\left(h_{p \vartheta_{(j)}}\right)_{m \times n}, Q$ and $w$ as input, the generalized WPHFAHMM operator (Suppose $Q=(1,1,1,1), \theta=3$ ) is used to calculate the overall preference value of each alternative. For example, the comprehensive of $Z_{1}$ is

$$
\begin{aligned}
& \operatorname{WPHFAHMM}{ }^{Q}\left(h_{1 p}, h_{2 p}, h_{3 p}, h_{4 p}\right) \\
& =\left(\frac{1}{4 !}\left(\bigoplus_{\vartheta \in S_{4}} \bigotimes_{j=1}^{4}\left(4 w_{p \vartheta_{(j)}} h_{p \vartheta_{(j)}}\right)^{q_{j}}\right)\right)^{\frac{1}{\sum_{j=1}^{4} q_{j}}} \\
& =\bigcup_{\left(\gamma_{j}, \eta_{j}\right) \in\left(\mu_{j}, v_{j}\right)} \sqrt{\frac{\left(v_{1}+\left(\theta^{2}-1\right) v_{2}\right)^{\lambda}-\left(v_{1}-v_{2}\right)^{\lambda}}{\left(u_{1}+\left(\theta^{2}-1\right) u_{2}\right)^{\lambda}+(\theta-1)\left(u_{1}-u_{2}\right)^{\lambda}}}, \\
& \text { where } \lambda=\frac{1}{\left(v_{1}+\left(\theta^{2}-1\right) v_{2}\right)^{\lambda}+(\theta-1)\left(v_{1}-v_{2}\right)^{\lambda}}, \\
& u_{1}=\prod_{\vartheta \in S_{4}}\left(\prod_{j=1}^{4}\left(\left(\theta+(1-\theta)\left(1-\gamma_{\theta(j)}^{2}\right)\right)^{4 w_{j}}+\left(\theta^{2}-1\right)\left(1-\gamma_{\theta(j)}^{2}\right)^{n w_{j}}\right)^{q_{j}}+\right. \\
& \left.\left(\theta^{2}-1\right) \prod_{j=1}^{n}\left(\left(\theta+(1-\theta)\left(1-\gamma_{\theta(j)}^{2}\right)\right)^{4 w_{j}}-\left(1-\gamma_{\theta(j)}^{2}\right)^{4 w_{j}}\right)^{q_{j}}\right)^{\frac{1}{4 !}},
\end{aligned}
$$

$$
\begin{aligned}
& u_{2}=\prod_{\vartheta \in S_{4}}\left(\prod_{j=1}^{4}\left(\left(\theta+(1-\theta)\left(1-\gamma_{\theta(j)}^{2}\right)\right)^{4 w_{j}}+\left(\theta^{2}-1\right)\left(1-\gamma_{\theta(j)}^{2}\right)^{4 w_{j}}\right)^{q_{j}}-\right. \\
& \left.\prod_{j=1}^{4}\left(\left(\theta+(1-\theta)\left(1-\gamma_{\theta(j)}^{2}\right)\right)^{4 w_{j}}-\left(1-\gamma_{\theta(j)}^{2}\right)^{4 w_{j}}\right)^{q_{j}}\right)^{\frac{1}{4 !}}, \\
& v_{1}=\prod_{\vartheta \in S_{4}}\left(\prod_{j=1}^{4}\left(\left(\theta+(1-\theta) \eta_{\theta(j)}^{2}\right)^{4 w_{j}}+\left(\theta^{2}-1\right) \eta_{\theta(j)}^{4 w_{j}}\right)^{q_{j}}+\right. \\
& \left.\left(\theta^{2}-1\right) \prod_{j=1}^{4}\left(\left(\theta+(1-\theta) \eta_{\theta(j)}^{2}\right)^{4 w_{j}}-\eta_{\theta(j)}^{8 w_{j}}\right)^{q_{j}}\right)^{\frac{1}{4 !}}, \\
& v_{2}=\prod_{\vartheta \in S_{4}}\left(\prod_{j=1}^{4}\left(\left(\theta+(1-\theta) \eta_{\theta(j)}^{2}\right)^{4 w_{j}}+\left(\theta^{2}-1\right) \eta_{\theta(j)}^{8 w_{j}}\right)^{q_{j}}-\right. \\
& \left.\prod_{j=1}^{4}\left(\left(\theta+(1-\theta) \eta_{\theta(j)}^{2}\right)^{4 w_{j}}-\eta_{\theta(j)}^{8 w_{j}}\right)^{q_{j}}\right)^{\frac{1}{4 !}} .
\end{aligned}
$$

Then

$$
\begin{aligned}
& \operatorname{WPHFAHMM}^{Q}\left(h_{1 p}, h_{2 p}, h_{3 p}, h_{4 p}\right) \\
& =\left\{\begin{array}{l}
\{(0.2,0.3),(0.3,0.4)\}, \\
\{(0.4,0.6),(0.5,0.4),(0.7,0.2)\} \\
\{(0.4,0.5),(0.6,0.3)\}, \\
\{(0.6,0.3),(0.7,0.4)\}
\end{array}\right\} \\
& =\left\{\begin{array}{l}
\{0.357468,0.374823,0.398696,0.417558, \ldots, \\
0.457773,0.478537,0.506760,0.528819\}, \\
\{0.527741,0.543744,0.491898,0.508755, \ldots, \\
0.467861,0.485401,0.427919,0.446677\}
\end{array}\right\}
\end{aligned}
$$

Similarly, the comprehensive values of the remaining alternatives are calculated.

Step 3. Calculate the scores and accuracies. The accuracy and score values of each alternative are respectively calculated using Equation (6) and Equation (7). The calculated results are shown in the Table 4.

Step 4. Generate a sort of all alternatives. Based on Table 4, the ranking method in Definition 4 is applied to generate a sort of the five alternatives. The generated sort is $z_{3}>z_{4}>z_{5}>z_{2}>z_{1}$.

Table 3

Pythagorean hesitant fuzzy decision matrix

\begin{tabular}{ccc}
\hline Alternatives & $C_{1}$ & $C_{2}$ \\
\hline$Z_{1}$ & $\{(0.2,0.3),(0.3,0.4)\}$ & $\{(0.4,0.6),(0.5,0.4),(0.7,0.2)\}$ \\
$Z_{2}$ & $\{(0.4,0.3),(0.6,0.4)\}$ & $\{(0.5,0.6),(0.6,0.4)\}$ \\
$Z_{3}$ & $\{(0.6,0.2),(0.7,0.3)\}$ & $\{(0.5,0.3),(0.5,0.4)\}$ \\
$Z_{4}$ & $\{(0.6,0.5),(0.7,0.4)\}$ & $\{(0.5,0.2),(0.6,0.5)\}$ \\
$Z_{5}$ & $\{(0.3,0.3),(0.6,0.4)\}$ & $\{(0.5,0.4),(0.7,0.4)\}$ \\
\hline Alternatives & $C_{3}$ & $\{(0.6,0.3),(0.7,0.4)\}$ \\
\hline$Z_{1}$ & $\{(0.4,0.5),(0.6,0.3)\}$ & $\{(0.5,0.4),(0.7,0.6)\}$ \\
$Z_{2}$ & $\{(0.5,0.3),(0.5,0.6)\}$ & $\{(0.4,0.5),(0.6,0.4)\}$ \\
$Z_{3}$ & $\{(0.5,0.2),(0.8,0.6),(0.8,0.2)\}$ & $\{(0.6,0.2),(0.6,0.3),(0.8,0.4)\}$ \\
$Z_{4}$ & $\{(0.4,0.3),(0.5,0.4)\}$ & $\{(0.4,0.6),(0.5,0.3)\}$ \\
$Z_{5}$ & $\{(0.6,0.4),(0.7,0.4)\}$ &
\end{tabular}

Table 4

The calculated accuracy and score values

$\begin{array}{llllll}\text { Indicator } & Z_{1} & Z_{2} & Z_{3} & Z_{4} & Z_{5}\end{array}$




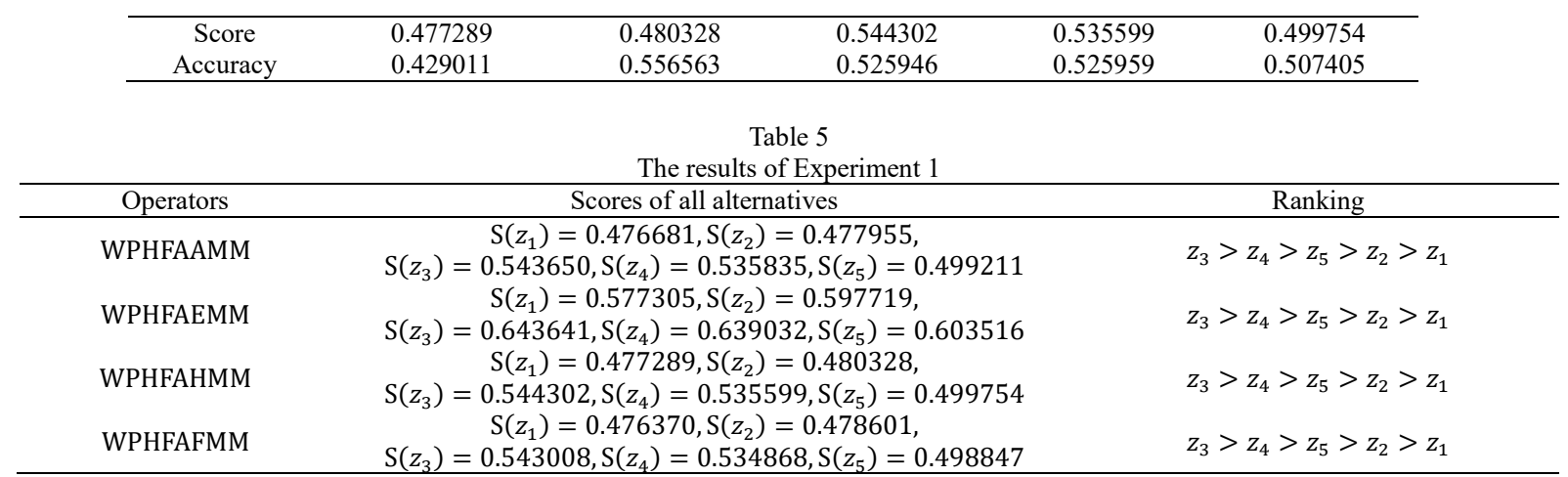

\subsection{Experiments}

To explore the effect of using different groups of aggregation operators and assigning different parameter values on the aggregation result, the following three experiments were carried out:

(1) Experiment 1 was conducted to show the effect of using different groups of operators on the aggregation result. In this experiment, $\left(h_{i j}\right)_{m \times n}, Q=$ $(1,1,1,1), \theta=3$ and $w=(0.4,0.1,0.2,0.3)^{T}$ were used as the input of the WPHFAMM operator.

The results of the experiment are the scores and rankings of the five alternatives, which are shown in Table 5. As can be seen from Table 5, the ranking results of the four operators are the same. This indicate that leveraging different groups of operators has no obvious impact on the aggregation result.

(2) Experiment 2 was carried out to show the effect of assigning different values to $\theta$ on the aggregation result. $\left(h_{i j}\right)_{m \times n}, Q=(1,1,1,1), \theta$ and $w=$ $(0.4,0.1,0.2,0.3)^{T}$ were used as the input of WPHFAHMM and WPHFAFMM operators. The value of the parameter $\theta$ was adapted from 1 to 32 . The scores obtained by the WPHFAHMM and WPHFAFMM operators are shown in Figure 1 and Figure 2, respectively.

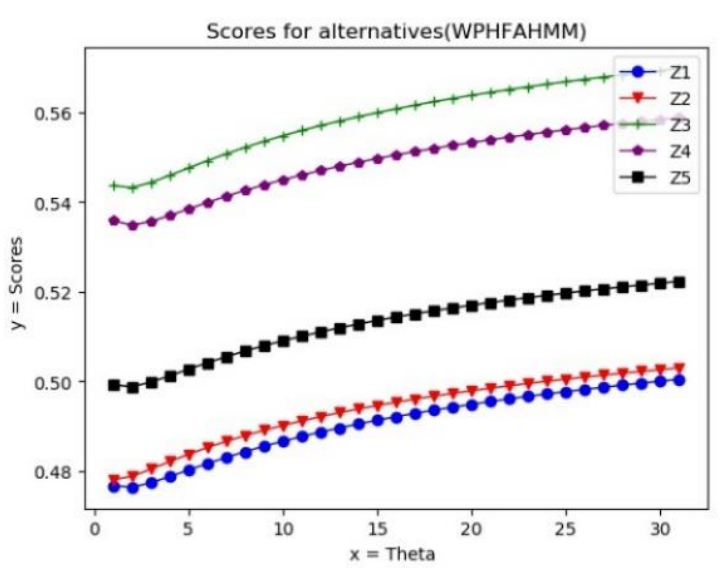

Fig.1. The scores of alternatives obtained by WPHFAHMM.

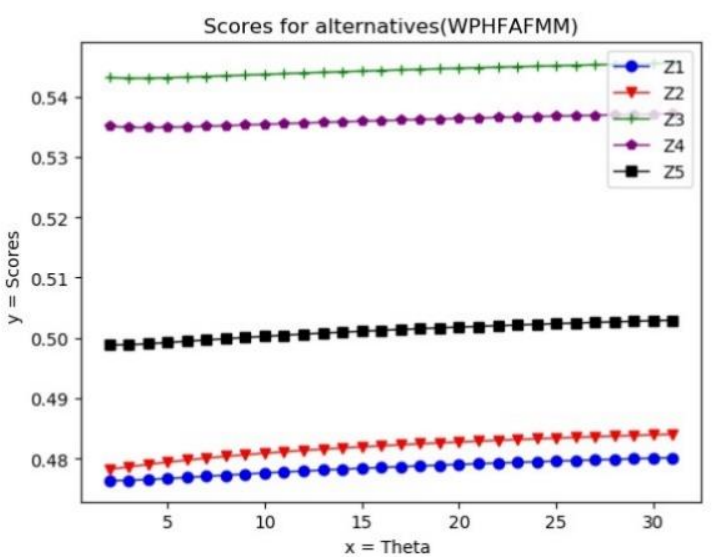

Fig.2. The scores of alternatives obtained by WPHFAFMM.

From Figure 1 and Figure 2, it can be observed that the score values increase as $\theta$ increases. The ranking orders of the two operators remain the same. This reveals that both the WPHFAHMM and WPHFAFMM 
operators are effective for solving the MCDM problems based on PHFNs.

It's worth noting that, as described in subsection 3.2 (Equation (26)), if the value of the parameter $\theta$ is assigned as 1 and 2, the WPHFAHMM operator will reduce to the WPHFAAMM and WPHFAEMM opera- tors, respectively. The score values and the sort of alternatives derived from the WPHFAAMM and WPHFAEMM operators are presented in Table 6 with the condition that $Q=(1,1,1,1)$ and $w=(0.4,0.1,0.2,0.3)^{T}$. From Table 6, it can be observed that the ranking orders of the two operators are the same, which is consistent with the result of Experiment 1.

Table 6

The results of WPHFAAMM and WPHFAEMM

\begin{tabular}{ccc}
\hline Operators & Scores of all alternatives & Ranking \\
\hline WPHFAAMM & $\mathrm{S}\left(z_{1}\right)=0.476681, \mathrm{~S}\left(z_{2}\right)=0.477955$, & $z_{3}>z_{4}>z_{5}>z_{2}>z_{1}$ \\
& $\mathrm{~S}\left(z_{3}\right)=0.543650, \mathrm{~S}\left(z_{4}\right)=0.535835, \mathrm{~S}\left(z_{5}\right)=0.499211$ & \\
WPHFAEMM & $\mathrm{S}\left(z_{1}\right)=0.476312, \mathrm{~S}\left(z_{2}\right)=0.478748$, & $z_{3}>z_{4}>z_{5}>z_{2}>z_{1}$ \\
\hline
\end{tabular}

Table 7

The results of Experiment 3. Note: $\theta=3$

\begin{tabular}{ccc}
\hline$Q$ & The ranking of WPHFAHMM & The ranking of WPHFAFMM \\
\hline$(3,0,0,0)$ & $z_{3}>z_{4}>z_{5}>z_{1}>z_{2}$ & $z_{3}>z_{4}>z_{5}>z_{1}>z_{2}$ \\
$(2,0,0,0)$ & $z_{3}>z_{4}>z_{5}>z_{1}>z_{2}$ & $z_{3}>z_{4}>z_{5}>z_{1}>z_{2}$ \\
$(1,0,0,0)$ & $z_{3}>z_{4}>z_{5}>z_{2}>z_{1}$ & $z_{3}>z_{4}>z_{5}>z_{2}>z_{1}$ \\
$(1,1,0,0)$ & $z_{3}>z_{4}>z_{5}>z_{1}>z_{2}$ & $z_{3}>z_{4}>z_{5}>z_{1}>z_{2}$ \\
$(1,1,1,0)$ & $z_{3}>z_{4}>z_{5}>z_{1}>z_{2}$ & $z_{3}>z_{4}>z_{5}>z_{1}>z_{2}$ \\
$(1,1,1,1)$ & $z_{3}>z_{4}>z_{5}>z_{2}>z_{1}$ & $z_{3}>z_{4}>z_{5}>z_{2}>z_{1}$ \\
$(0.25,0.25,0.25,0.25)$ & $z_{3}>z_{4}>z_{5}>z_{2}>z_{1}$ & $z_{3}>z_{4}>z_{5}>z_{2}>z_{1}$ \\
\hline
\end{tabular}

(3) Experiment 3 was carried out to show the effect of assigning different values to $Q$ on aggregation result. $\left(h_{i j}\right)_{m \times n}, Q, \theta=3$ and $w=(0.4,0.1,0.2,0.3)^{T}$ were used as the input of the WPHFAHMM and WPHFAFMM operators. There are seven groups of values of $Q$. The results of the two operators are shown in Table 7.

In Table 7, when $Q=(3,0,0,0), Q=(2,0,0,0), Q=$ $(1,1,0,0)$ and $Q=(1,1,1,0)$, the rankings are $\mathrm{z}_{3}>\mathrm{z}_{4}>$ $\mathrm{z}_{5}>\mathrm{z}_{1}>\mathrm{z}_{2}$. When $Q=(1,0,0,0), Q=(1,1,1,1)$ and $Q=$ $(0.25,0.25,0.25,0.25)$, the rankings are $\mathrm{z}_{3}>\mathrm{z}_{4}>\mathrm{z}_{5}>\mathrm{z}_{2}>$ $Z_{1}$. Though the rankings change slightly, the best alternatives remain the same (i.e., $\left.Z_{3}\right)$. When $\mathrm{Q}=(1,0,0,0)$, it's worth noting that WPHFAMM operator reduces to
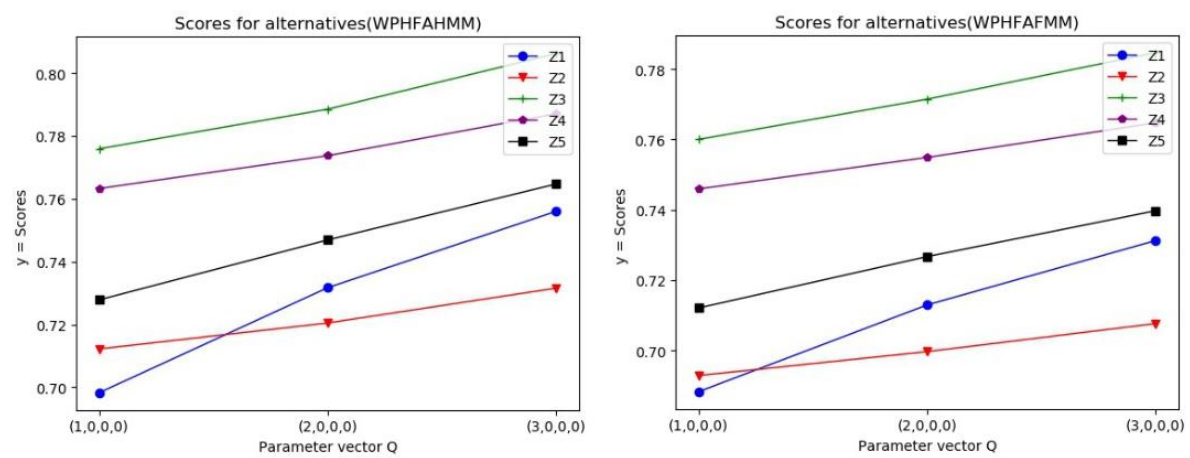

Fig.3. Scores of alternatives for different parameters $Q$. the APHFWA operator [35].

In addition, Figure 3 and Figure 4 draw our attention to the diversification of the parameters $Q$ in more details: 1) For the WPHFAHMM and WPHFAFMM operators with the condition that $\theta=3$, the larger the real number of $Q$, the greater the value of the score function when $Q$ has merely one real number and the rest are 0; 2) For the WPHFAHMM and WPHFAFMM operators with the condition that $\theta=3$, the more complex relationships among criteria, the greater the value of the score function when $Q$ has more than one real number. Based on this, decision makers can choose different values of $Q$ according to the actual relationships of criteria. 

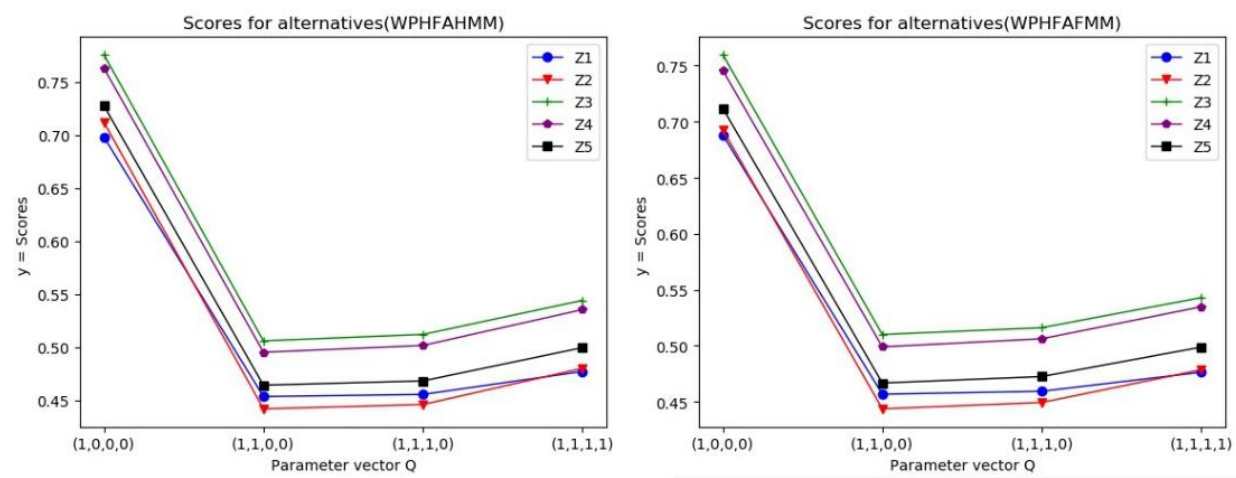

Fig.4. Scores of alternatives for different parameters $Q$.

\subsection{Comparisons}

So far, a number of MCDM methods based on the operators that can aggregate PHFNs have been proposed. In this subsection, these MCDM methods are compared with the developed method in this paper. Specifically, the comparison methods include the methods based on the Pythagorean hesitant fuzzy Hamacher ordered weighted averaging (PHFHOWA) and Pythagorean hesitant fuzzy Hamacher ordered weighted (PHFHOWG) operators [30], the Pythagorean hesitant fuzzy Hamacher weighted averaging (PHFHWA) and
Pythagorean hesitant fuzzy Hamacher weighted geometric (PHFHWG) operators [35], the weighted Pythagorean hesitant fuzzy Maclaurin symmetric mean (WHPFMSM) operator [31], the Pythagorean hesitant fuzzy Hybrid weighted averaging (PHFHWA) and Pythagorean hesitant fuzzy Hybrid weighted geometric (PHFHWG) operators [33], and the presented WPHFAMM operator. A qualitative comparison and a quantitative comparison among these methods are carried out to verify the effectiveness and show the advantages of the proposed method.

Table 8

The results of the qualitative comparisons of different methods

\begin{tabular}{|c|c|c|c|}
\hline Methods & Has desirable generality & Capture the interrelationships & Has desirable flexibility \\
\hline PHFHOW $A^{[30]}$ & $\times$ & $\times$ & $\times$ \\
\hline PHFHOWG ${ }^{[30]}$ & $\times$ & $x$ & $x$ \\
\hline PHFHW $A^{[35]}$ & $\times$ & $\times$ & $\times$ \\
\hline PHFHWG ${ }^{[35]}$ & $\times$ & $x$ & $x$ \\
\hline WHPFMSM ${ }^{[31]}$ & $\sqrt{ }$ & $\sqrt{ }$ & $\sqrt{ }$ \\
\hline PHFHWA $A^{[33]}$ & $\times$ & $x$ & $x$ \\
\hline PHFHW $G^{[33]}$ & $x$ & $x$ & $x$ \\
\hline WPHFAMM & $\sqrt{ }$ & $\sqrt{ }$ & $\sqrt{ }$ \\
\hline
\end{tabular}

Table 9

The results of the quantitative comparison of different methods

\begin{tabular}{ccc}
\hline Operator & Parameters & Ranking \\
\hline PHFHOWA $^{[30]}$ & $\gamma=3$ & $z_{3}>z_{4}>z_{5}>z_{1}>z_{2}$ \\
PHFHOWG $^{[30]}$ & $\gamma=3$ & $z_{3}>z_{4}>z_{5}>z_{2}>z_{1}$ \\
PHFHWA $^{[35]}$ & $\theta=3$ & $z_{3}>z_{4}>z_{2}>z_{5}>z_{1}$ \\
PHFHWG $^{[35]}$ & $\theta=3$ & $z_{3}>z_{4}>z_{1}>z_{5}>z_{2}$ \\
WHPFMSM $^{[31]}$ & $k=n$ & $z_{3}>z_{4}>z_{2}>z_{5}>z_{1}$ \\
PHFHWA $^{[33]}$ & - & $z_{3}>z_{4}>z_{5}>z_{1}>z_{2}$ \\
PHFHWG $^{[33]}$ & - & $z_{3}>z_{4}>z_{5}>z_{2}>z_{1}$ \\
WPHFAHMM & $z_{3}>z_{4}>z_{5}>z_{2}>z_{1}$ \\
WPHFAFMM & $Q=(1,1,1,1), \theta=3$ & $z_{3}>z_{4}>z_{5}>z_{2}>z_{1}$ \\
\hline
\end{tabular}




\subsubsection{Qualitative comparison}

This comparison is carried out by comparing the characteristics of the selected methods. The comparable characteristics are the generality of aggregation operators, whether takes into account the interrelationships of different criteria and the flexibility of aggregation operators. The comparison results are shown in Table 8 . The details of the comparison are explained as follows:

(1) Generality. The PHFHOWA and PHFHOWG method [30], the PHFHWA and PHFHWG method [35], and the PHFHWA and PHFHWG method [33] are based on a specific type of ATT. The WHPFMSM method [31] and the proposed method are based on any types of ATTs. Therefore, the WHPFMSM and proposed methods are more general than the remaining methods. Further, the proposed method provides more satisfying generality than the WHPFMSM method since the MSM operator is a special case of the MM operator.

(2) Interrelationships. Among the comparison methods, the WHPFMSM and proposed methods have the capability to deal with the interrelationships of different criteria in MCDM problems because they respectively use the MSM and MM operators.

(3) Flexibility. Since any types of ATTs can provide flexible parameters, the WHPFMSM and proposed methods are more flexible than the remaining methods.

As can be summarized from the qualitative comparison, the proposed method not only has desirable generality and flexibility, but also can consider the interrelationships of criteria more comprehensively.

\subsubsection{Quantitative comparison}

The quantitative comparison is carried out based on the numerical example in subsection 5.1. In this comparison, the PHFHOWA [30], PHFHOWG [30], PHFHWA [35], PHFHWG [35], WHPFMSM [31], PHFHWA [33], PHFHWA [33] and proposed methods are applied to resolve the MCDM problem in the example. The results of the experiment are the rankings of the five alternatives, which are shown in Table 9.

As can be seen from Table 9, the first two optimal alternatives derived from all comparison methods are $z_{3}$ and $z_{4}$, which demonstrate the feasibility and effectiveness of the proposed method. Careful readers may find that the ranking of the proposed method is slightly different at the third, fourth and fifth places with the rankings of the PHFHWA, PHFHWG, WHPFMSM and PHFHWA methods. The reason is that different methods use different aggregation operators and have different mathematical properties.

\section{Conclusion}

The ATT can generate versatile and flexible operational laws for fuzzy numbers, while the MM operator is capable of capturing the interrelationships of aggregated arguments. In this paper, the MM operator and the ATT have been combined to present a PHFAMM operator and a WPHFAMM operator, and a MCDM method based on these operators has been proposed. To be more specific, the generalised and specific expressions of the two operators have been constructed and their properties have been explored and proved. The paper has also reported a practical example, a set of experiments, and qualitative and quantitative comparisons to demonstrate the proposed method. The demonstrate results suggest that the method is feasible and effective that can provide desirable generality and flexibility in aggregation of criterion values and capturing of criterion relationships.

In future work, the application of the proposed method in real decision-making problems will be studied. The application of the similarity measures of the presented operators in knowledge graphs is a representative example that will be studied. Further, other types of aggregation operators can easily be derived by combining the MM operator with dual hesitant Pythagorean fuzzy information and ATT or the triangle intuitionistic fuzzy information and picture fuzzy information. The development of 2-tuple linguistic neutrosophic fuzzy Archimedean MM operators may also be explored. For all of these studies, the research findings in references [32,48-52] could be helpful.

\section{References}

[1] Figueira J, Greco S, Ehrgott M. Multiple Criteria Decision Analysis: State of the Art Surveys[M]. Springer New York, 2005.

[2] Zadeh LA. Fuzzy Sets[J]. Information \& Control, 1965, 8(3): 338-353.

[3] Atanassov KT. Intuitionistic fuzzy sets[J]. Fuzzy Sets \& Systems, 1986, 20(1): 87-96.

[4] Atanassov KT. More on intuitionistic fuzzy sets[J]. 1989, 33(1): 37-45.

[5] Atanassov KT. Two theorems for intuitionistic fuzzy sets[J]. Fuzzy Sets and Systems, 2000, 110(2): 267-269.

[6] Garg H, Rani D. Complex interval-valued intuitionistic fuzzy sets and their aggregation operators[J]. Fundamenta Informaticae, 2019, 164(1): 61-101.

[7] Garg H, Rani D. A robust correlation coefficient measure of complex intuitionistic fuzzy sets and their applications in decision-making[J]. Applied Intelligence, 2019, 49(2): 496-512.

[8] Yager RR. Pythagorean fuzzy subsets[C]. IFSA World Congress and NAFIPS Annual Meeting (IFSA/NAFIPS), 2013. IEEE.

[9] Yager RR. Pythagorean Membership Grades in Multicriteria 
Decision Making[J]. IEEE Transactions on Fuzzy Systems, 2014, 22(4): 958-965.

[10] Zhang X, Xu Z. Extension of TOPSIS to Multiple Criteria Decision Making with Pythagorean Fuzzy Sets[J]. International Journal of Intelligent Systems, 2014, 29(12): 1061-1078.

[11] Garg H. A New Generalized Pythagorean Fuzzy Information Aggregation Using Einstein Operations and Its Application to Decision Making[J]. International Journal of Intelligent Systems, 2016, 31(9): 886-920.

[12] Garg H. Generalized Pythagorean fuzzy geometric aggregation operators using Einstein t-norm and t-conorm for multicriteria decision-making process[J]. International Journal of Intelligent Systems, 2017, 32(6): 597-630

[13] Garg H. Generalised Pythagorean fuzzy geometric interactive aggregation operators using Einstein operations and their application to decision making[J]. Journal of Experimental \& Theoretical Artificial Intelligence, 2018, 30(6): 763-794.

[14] Garg H. A novel correlation coefficients between Pythagorean fuzzy sets and its applications to decision-making processes $[\mathrm{J}]$ International Journal of Intelligent Systems, 2016, 31(12): 1234-1252.

[15] Gou X, Xu Z, Ren P. The Properties of Continuous Pythagorean Fuzzy Information[J]. International Journal of Intelligent Systems, 2016, 31(5): 401-424.

[16] Peng X, Selvachandran G. Pythagorean fuzzy set: state of the art and future directions[J]. Artificial Intelligence Review, 2017, https://doi.org/10.1007/s10462-017-9596-9.

[17] Garg H. Linguistic Pythagorean fuzzy sets and its applications in multiattribute decision-making process $[\mathrm{J}]$. International Journal of Intelligent Systems, 2018, 33(6): 1234-1263.

[18] Wei G, Lu M. Pythagorean fuzzy power aggregation operators in multiple attribute decision making[J]. International Journal of Intelligent Systems, 2018, 33(1): 169-186.

[19] Wei G, Garg H, Gao H, et al. Interval-valued Pythagorean fuzzy Maclaurin symmetric mean operators in multiple attribute decision making[J]. Ieee Access, 2018, 6: 67866-67884.

[20] Garg H. New exponential operational laws and their aggregation operators for interval-valued Pythagorean fuzzy multicriteria decision-making[J]. International Journal of Intelligent Systems, 2018, 33(3): 653-683.

[21] Garg H. New logarithmic operational laws and their aggregation operators for Pythagorean fuzzy set and their applications[J]. International Journal of Intelligent Systems, 2019, 34(1): 82-106.

[22] Peng X, Li W. Algorithms for interval-valued Pythagorean fuzzy sets in emergency decision making based on multiparametric similarity measures and WDBA[J]. IEEE Access, 2019, 7: 7419-7441.

[23] Peng X. Algorithm for Pythagorean fuzzy multi-criteria decision making based on WDBA with new score function[J]. Fundamenta Informaticae, 2019, 165(2): 99-137.

[24] Torra V, Narukawa Y. On hesitant fuzzy sets and decision[C]. IEEE International Conference on Fuzzy Systems. 2009, IEEE.

[25] Torra V. Hesitant fuzzy sets[J]. International Journal of Intelligent Systems, 2010, 25(6): 529-539.

[26] Qin J, Liu X, Pedrycz W. Frank aggregation operators and their application to hesitant fuzzy multiple attribute decision making[J]. Applied Soft Computing, 2016, 41: 428-452.

[27] Liang D, Xu Z. The new extension of TOPSIS method for multiple criteria decision making with hesitant Pythagorean fuzzy sets[J]. Applied Soft Computing, 2017, 60: 167-179.

[28] Liu P, Wang P. Multiple-attribute decision-making based on Archimedean Bonferroni Operators of q-rung orthopair fuzzy numbers[J]. IEEE Transactions on Fuzzy systems, 2019, 27(5): 834-848.

[29] Garg H. Hesitant Pythagorean fuzzy sets and their aggregation operators in multiple attribute decision-making[J]. International Journal for Uncertainty Quantification, 2018, 8(3): $267-$ 289.

[30] Wei G, Lu M. Pythagorean Hesitant Fuzzy Hamacher Aggregation Operators in Multiple-Attribute Decision Making[J]. Journal of Intelligent Systems, 2017, https://doi.org/10.1515/ jisys-2017-0106.

[31] Garg H. Hesitant Pythagorean fuzzy Maclaurin symmetric mean operators and its applications to multi-attribute decisionmaking process. International Journal of Intelligent Systems, 2019, 34(4): 601-626.

[32] Tang X, Wei G. Multiple attribute decision-making with dual hesitant Pythagorean fuzzy information[J]. Cognitive Computation, 2019, 11(2): 193-211.

[33] Khan MSA, Abdullah S, Ali A, et al. Hybrid aggregation operators based on Pythagorean hesitant fuzzy sets and their application to group decision making[J]. Granular Computing, 2019, 4(3): 469-482.

[34] Gao H, Wei G, Huang Y. Dual Hesitant Bipolar Fuzzy Hamacher Prioritized Aggregation Operators in Multiple Attribute Decision Making[J]. IEEE Access, 2018: 11508-11522.

[35] Sarkar A, Biswas A. Multicriteria decision-making using Archimedean aggregation operators in Pythagorean hesitant fuzzy environment[J]. International Journal of Intelligent Systems, 2019, 34(7): 1361-1386.

[36] Nguyen HT, Walker CL, Walker EA. A first course in fuzzy logic[M]. 2018, CRC Press.

[37] Yager RR, Abbasov AM. Pythagorean Membership Grades, Complex Numbers, and Decision Making $[\mathrm{J}]$. International Journal of Intelligent Systems, 2013, 28(5): 436-452.

[38] Simon D. Fuzzy Sets and Fuzzy Logic: Theory and Applications $[J]$. Control Engineering Practice, 1996, 4(9): 1332-1333.

[39] Peng X, Yang Y. Pythagorean fuzzy Choquet integral based MABAC method for multiple attribute group decision making[J]. International Journal of Intelligent Systems, 2016, 31(10): 989-1020

[40] Muirhead RF. Some methods applicable to identities and inequalities of symmetric algebraic functions of n letters[J]. Proceedings of the Edinburgh Mathematical Society, 1902, 21: 144-162.

[41] Bonferroni C. Sulle medie multiple di potenze. Bollettino dell'Unione Matematica Italiana 1950, 5: 267-270.

[42] Detemple DW, Robertson JM. On generalized symmetric means of two varibles[J]. Angewandte Chemie, 1979, 47(25): 4638-4660.

[43] McLaurin C. A second letter to Martin Folges, Esq. concerning the roots of equations with the demonstration of other rules in algebra[J]. Philos. Trans. R. Soc. Lond, 1730, 1730(36): 59-96.

[44] Hong Z, Rong Y, Qin Y, et al. Hesitant fuzzy dual Muirhead mean operators and its application to multiple attribute decision making[J]. Journal of Intelligent \& Fuzzy Systems, 2018, 35(2): 2161-2172.

[45] Liu P, Li Y, Zhang M, et al. Multiple-attribute decision-making method based on hesitant fuzzy linguistic Muirhead mean aggregation operators[J]. Soft Computing, 2018, 22(16): 55135524.

[46] Wang J, Zhang R, Zhu X, et al. Some hesitant fuzzy linguistic Muirhead means with their application to multiattribute group decision-making[J]. Complexity, 2018, 5087851.

[47] Zhu J, Li Y. Pythagorean fuzzy Muirhead mean operators and their application in multiple-criteria group decision-Making[J] Information, 2018, 9(6): 142

[48] Liang D, Zhang Y, Xu Z, et al. Pythagorean fuzzy Bonferroni mean aggregation operator and its accelerative calculating algorithm with the multithreading[J]. International Journal of Intelligent Systems, 2018, 33(3): 615-633. 
[49] Liang D, Darko A P, Xu Z, et al. The linear assignment method for multicriteria group decision making based on interval valued Pythagorean fuzzy Bonferroni mean[J]. International Journal of Intelligent Systems, 2018, 33(11): 2101-2138.

[50] Wang J, Gao H, Wei G. Some 2-tuple linguistic neutrosophic number Muirhead mean operators and their applications to multiple attribute decision making[J]. Journal of Experimental \& Theoretical Artificial Intelligence, 2019, 31(3): 409-439.

[51] Wang J, Gao H, Wei G. The generalized Dice similarity measures for Pythagorean fuzzy multiple attribute group decision making[J]. International Journal of Intelligent Systems, 2019, 34(6): 1158-1183.

[52] Peng S. Study on enterprise risk management assessment based on picture fuzzy multiple attribute decision-making method[J]. Journal of Intelligent \& Fuzzy Systems, 2017, 33(6): 3451-3458. 